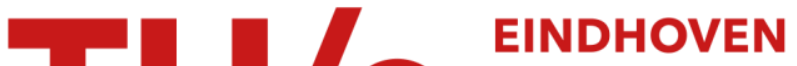 UNIVERSITY OF TECHNOLOGY
}

\section{Direct numerical simulation of fluid flow and dependently coupled heat and mass transfer in fluid-particle systems}

\section{Citation for published version (APA):}

Lu, J., Peters, E. A. J. F., \& Kuipers, J. A. M. (2019). Direct numerical simulation of fluid flow and dependently coupled heat and mass transfer in fluid-particle systems. Chemical Engineering Science, 204, 203-219. https://doi.org/10.1016/j.ces.2019.02.043

\section{Document license:}

TAVERNE

DOI:

10.1016/j.ces.2019.02.043

Document status and date:

Published: 31/08/2019

\section{Document Version:}

Publisher's PDF, also known as Version of Record (includes final page, issue and volume numbers)

\section{Please check the document version of this publication:}

- A submitted manuscript is the version of the article upon submission and before peer-review. There can be important differences between the submitted version and the official published version of record. People interested in the research are advised to contact the author for the final version of the publication, or visit the $\mathrm{DOI}$ to the publisher's website.

- The final author version and the galley proof are versions of the publication after peer review.

- The final published version features the final layout of the paper including the volume, issue and page numbers.

Link to publication

\section{General rights}

Copyright and moral rights for the publications made accessible in the public portal are retained by the authors and/or other copyright owners and it is a condition of accessing publications that users recognise and abide by the legal requirements associated with these rights.

- Users may download and print one copy of any publication from the public portal for the purpose of private study or research.

- You may not further distribute the material or use it for any profit-making activity or commercial gain

- You may freely distribute the URL identifying the publication in the public portal.

If the publication is distributed under the terms of Article 25fa of the Dutch Copyright Act, indicated by the "Taverne" license above, please follow below link for the End User Agreement:

www.tue.nl/taverne

Take down policy

If you believe that this document breaches copyright please contact us at:

openaccess@tue.nl

providing details and we will investigate your claim. 


\title{
Direct numerical simulation of fluid flow and dependently coupled heat and mass transfer in fluid-particle systems
}

\author{
Jiangtao Lu, E.A.J.F. Peters*, J.A.M. Kuipers \\ Multiphase Reactors Group, Department of Chemical Engineering and Chemistry, Eindhoven University of Technology, P.O. Box 513, 5600 MB Eindhoven, the Netherlands
}

\section{H I G H L I G H T S}

- Fully resolved simulations of fluidparticle systems.

- Surface reaction with significant heat effects.

- Temperature-dependent reaction rates determined by the Arrhenius equation.

- Simulation parameters adopted from realistic POX reaction.

\section{A R T I C L E I N F O}

\section{Article history:}

Received 26 November 2018

Received in revised form 21 January 2019

Accepted 16 February 2019

Available online 6 March 2019

\section{Keywords:}

Immersed boundary method

Gas-solid system

Surface reaction

Coupled heat and mass transfer

Damköhler number

Arrhenius equation

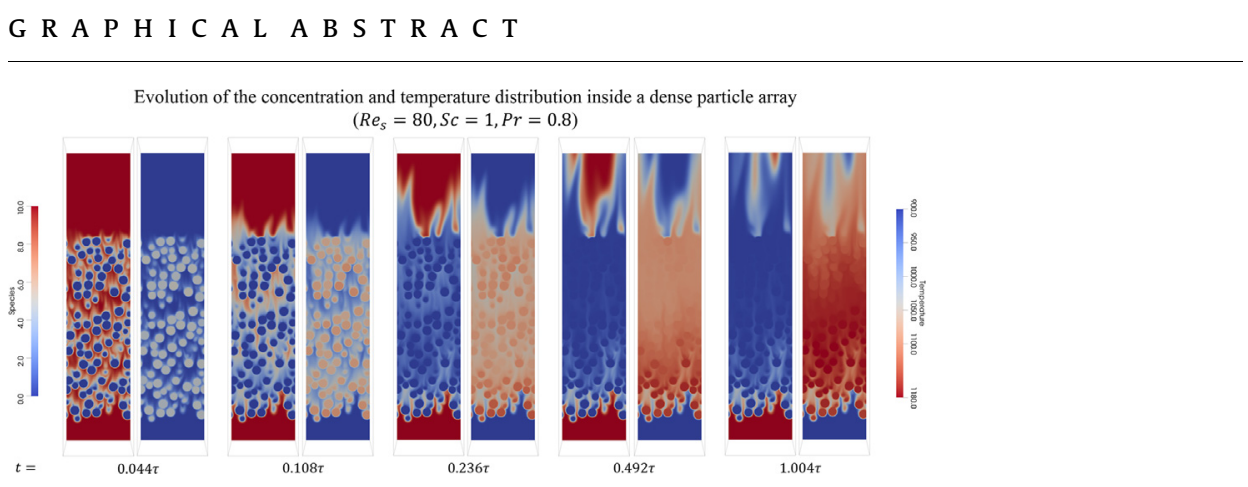

A B S T R A C T

In this paper, an efficient ghost-cell based immersed boundary method (IBM) is used to perform direct numerical simulation (DNS) of reactive fluid-particle systems. With an exothermic first order reaction proceeding at the exterior particle surface, the solid temperature rises and consequently increases the reaction rate via an Arrhenius temperature dependence. In other words, the heat and mass transport is dependently coupled through the particle thermal energy equation and the Arrhenius equation, and they offer dynamic boundary conditions for the fluid phase thermal energy equation and species equation respectively. The fluid-solid coupling is enforced at the exact position of the particle surface by implicit incorporation of the boundary conditions into the discretized momentum, species and thermal energy conservation equations of the fluid phase. Different fluid-particle systems are studied with increasing complexity: a single sphere, three spheres and a dense array consisting of hundreds of randomly generated particles. In these systems the mutual impacts between heat and mass transport processes are investigated.

() 2019 Elsevier Ltd. All rights reserved.

\section{Introduction}

Reactive fluid-particle systems are widely encountered and utilized in various processes, such as catalytic synthesis in chemical industry, coal combustion in energy industry and ore reduction in metallurgical industry. Two important factors need to be considered in such complex heterogeneous systems: variable reaction

\footnotetext{
* Corresponding author.

E-mail address: E.A.J.F.Peters@tue.nl (E.A.J.F. Peters).
}

rates and significant heat effects. They pose considerable challenges to the equipment design as well as the process performance. In such circumstances, fundamentally understanding the heat and mass transport processes as well as the fluid flow is of tremendous importance. In the last century, numerous correlations obtained from experiments have been proposed for heat and mass transfer between the fluid and the solid phase for both fixed and fluidized systems (Gunn, 1978; Wakao and Funazkri, 1978; Hughmark, 1980; Kumar and Fan, 1994; Kim and Kang, 1997; Wagner et al., 1997). Although utilization of these correlations is quick and convenient to obtain average heat and mass transfer rates, detailed 


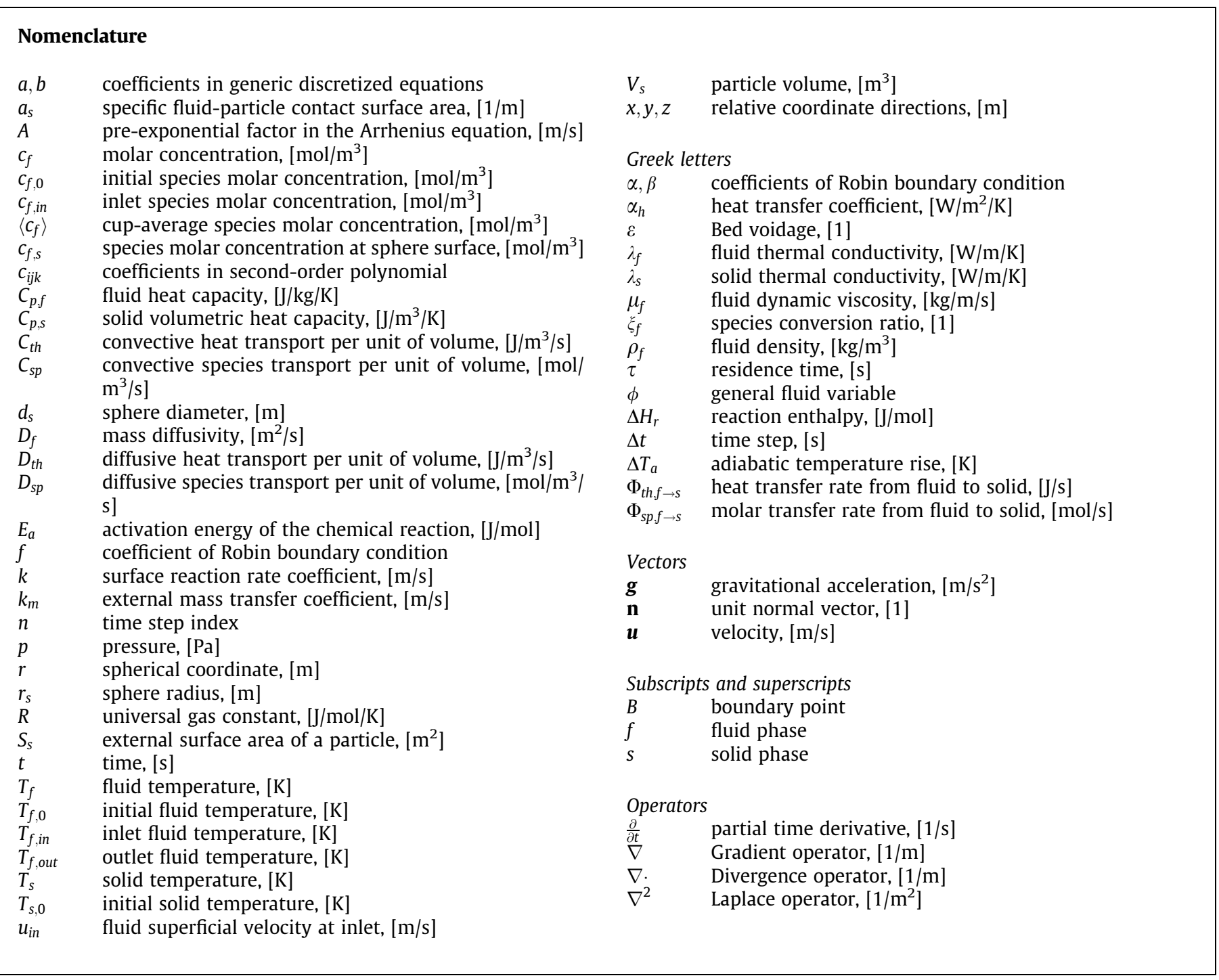

information is not easily quantified. For example, inhomogeneous particle distribution may lead to strongly irregular fluid behavior and consequently varying heat and mass transfer rates in both space and time. The complexity is further increased if the mass and heat transfer processes are mutually influenced. In other words, the heat effect associated with the mass transfer process will in turn influence the mass transfer process itself. The desired quantitative detailed information, however, can be produced using three-dimensional transient computational fluid dynamics (CFD), due to significant advances in algorithm development as well as computational power that have been made in the last few decades.

Due to the severe limitation of computational power, most of the earlier CFD work on reactive fluid-particle systems was limited to the usage of large scale models. Holloway and Sundaresan (2012) studied reacting gas-particle flows using coarse-grained two-fluid models. In their studies, constitutive relations for the filtered reaction rate and filtered species dispersion are postulated to close the filtered species balance equation, and effective reaction rates are used to rationalize the over-prediction in reactant conversion. Xue and Fox (2014) applied a kinetic theory based multi-fluid Eulerian model to study biomass gasification in fluidized bed gasifiers. Chemical kinetics and variable-particle-density model are coupled with the governing equations, and a time-splitting approach with fractional-time-step adaption is employed to maintain numerical stability of transport-reaction coupling. Zhuang et al. (2014) combined the discrete element method (DEM) with CFD to describe the gas-solid flow behavior in a fluidized bed reactor for methanol-to-olefins processes. The reaction is accounted for by incorporating the lumped kinetics into the gas phase, and the heat transfer is considered between particles as well as between gas and particles. Ku et al. (2015) proposed a CFD-DEM model to simulate biomass gasification in a fluidized bed reactor. Devolatilization, pyrolysis, char conversion, water vaporization and particle shrinkage are all considered for individual particles. Source terms resulting from the particle phase reactions as well as the reduced gas phase reactions are included in the gas phase governing equations.

With the rapid development of computational capability, DNS has emerged as a powerful tool to resolve all the details at the sub-particle level, and this provides fundamental insights in fluid-solid interactions. Such information can be further used to quantitatively evaluate microscale transport coefficients that are required as closures in coarser scale models. There are several DNS techniques proposed by pioneering researchers for modeling of fluid-particle systems such as the overset grid method (Chesshire and Henshaw, 1990), the arbitrary LagrangianEulerian method (Hu et al., 2001) and the Lattice-Boltzmann method (Ladd, 1994a, b), however in recent years the immersed boundary method (IBM) has received a lot of attention due to its considerable advantages of easy grid generation and efficient CPU 
utilization. IBM has been applied in various studies including elastic boundaries, complex geometries and moving objects (Fadlun et al., 2000; Kim et al., 2001; Takeuchi et al., 2010; Xu et al., 2013; Liu and Hu, 2014; Das et al., 2017). Another merit of IBM, utilized in this paper, is its easy addition of species and thermal energy equations following the fluid flow equations. They are then solved by using the same methodology. There are mainly two classes of IBM, specifically the continuous forcing method, i.e., CFM, and the discrete forcing method, i.e., DFM. CFM represents the immersed object by Lagrangian marker points, and introduces a forcing term determined by fulfilling the expected boundary condition in an interpolated manner into the governing equations to represent the fluid-solid interaction (Peskin, 1977; Goldstein et al., 1993; Saiki and Biringen, 1996; Uhlmann, 2005; Vanella and Balaras, 2009; Di and Ge, 2015). DFM calculates virtual variable values at ghost cells (i.e., cells inside the solid phase but required for solving the fluid phase equations) by extrapolating fluid variables near the interface together with the enforced boundary condition, to account for the influence of the immersed object (Mohd-Yusof, 1997; Udaykumar et al., 2001; Tseng and Ferziger, 2003; Marella et al., 2005; Ghias et al., 2007; Mittal et al., 2008). DFM is implemented in our DNS model, due to its considerable merit of treating the immersed boundary as a sharp interface and maintaining the same numerical stability conditions.

Contrary to intensive hydrodynamic studies, i.e., interfacial momentum exchange, in fluid-particle systems using IBM, much less attention was paid to the field of mass and heat transfer. Mass transfer studies have been reported by, e.g.: Deen and Kuipers (2013) applying DFM to dense fluid-particle systems assuming infinitely fast surface reaction and Gong et al. (2014) applying CFM to permeable moving interfaces to describe their deformability and interaction with the surrounding fluids. A somewhat larger amount of studies regarding heat transfer have been reported by, e.g.: Feng and Michaelides (2009) applying CFM to particulate flows assuming constant temperature at the sphere surface, Tenneti et al. (2013) applying CFM to account for the thermal interaction between the fluid and the isothermal particles in a statistically homogeneous particle domain and Xia et al. (2015) applying DFM to study the impact of natural convection on the cooling process of a freely settling sphere. In the scope of coupled heat and mass transfer very few IBM computational results are available. Deen and Kuipers (2014) extended their preceding DFM model to chemical reaction with heat production in a random array of stationary particles. Abdelsamie et al. (2016), Validi et al. (2017) and Boukharfane et al. (2018) studied compressible reactive multicomponent flows in combustion processes, with the source terms included in the conservation equations and IBM implemented to enforce the zero-gradient boundary condition for complex engine geometries or moving fuel particles. Some researchers reported particle-resolved numerical simulations for reactive fluid-particle systems without using IBM. Wehinger et al. (2015) studied dry reforming of methane in catalytic fixed bed reactors. 113 spheres were generated by DEM and detailed reaction mechanisms were applied. The boundary condition assumes that the produced/consumed species at the catalyst surface by desorption/adsorption equals the diffusive mass flux from/to the catalyst. Dixon (2017) studied steam methane reforming in a random packed bed inside a slender tube. The fluid flow field is fully coupled to the temperature and species distributions inside the catalyst through the solid particle method. In this method, the conservation equations in both fluid and solid phases are solved and the interfacial coupling is provided. Schulze et al. (2017) studied char conversion processes in fixed bed gasifiers. The species net production and the total reaction heat are directly incorporated into the governing equations. The species concentration and temperature on the particle surface, i.e., boundary conditions, are evaluated by balancing the transport and reactions.
In this paper, we extend an earlier reported DNS model (Lu et al., 2018a; Lu et al., 2018b; Lu et al., 2018c; Lu et al., 2018d) to simulate coupled heat and mass transfer processes in reactive fluid-solid systems. With our ghost-cell based immersed boundary method, the fluid-solid coupling is realized by a second order quadratic interpolation scheme. In this method, the Robin boundary condition is enforced exactly at the particle surface and implicitly at the level of discretized equations. A surface reaction rate is incorporated into the Robin boundary condition to describe the interplay between chemical transformation and mass transport in fluid-particle systems, while the Robin boundary condition reduces to the Dirichlet boundary condition with the value of the particle temperature. It should be noted that the heat and mass transfer are fully coupled in our DNS model. In other words, the solid temperature is determined by the thermal energy equation of individual particles and offers a dynamic boundary condition for the fluid phase thermal energy equation, while the surface reaction rate of each particle is updated through the Arrhenius equation and serves as a dynamic boundary condition for the fluid phase species equation.

We organize the current paper as follows. Following the description of our DNS model, the numerical details are presented. After that, the main results of different fluid-particle systems are reported including a single sphere, three spheres and a dense array. The conclusions are presented in the last section.

\section{Model description}

For the DNS model introduced for the current research, we assume: (1) The fluid phase is incompressible and Newtonian, and the solid phase is spherical catalyst particles; (2) Both fluid and solid phases have constant physical properties; (3) Diffusion follows Fick's laws; (4) Only the external particle surface is reactive, and the Biot number is much smaller than the unity so that individual particles have a uniform temperature. In our case, the Biot number is defined as:

$B i=\frac{\alpha_{h} d_{s}}{\lambda_{s}}$

where $\alpha_{h}$ is the convective heat transfer coefficient, $d_{s}$ is the particle diameter and $\lambda_{s}$ is the thermal conductivity of the solid phase. For small catalyst particles that are commonly composed of highly conductive metals (metal oxides), this assumption is valid.

\subsection{Governing equations}

The governing equations to describe the velocity, concentration and temperature distribution in the fluid phase are the NavierStokes, the species and the thermal energy equations, respectively given by:

$\nabla \cdot \boldsymbol{u}=0$

$\frac{\partial \rho_{f} \boldsymbol{u}}{\partial t}+\nabla \cdot\left(\rho_{f} \boldsymbol{u} \boldsymbol{u}\right)=-\nabla p+\mu_{f} \nabla^{2} \boldsymbol{u}+\rho_{f} \mathbf{g}$

$\frac{\partial c_{f}}{\partial t}+\nabla \cdot\left(c_{f} \boldsymbol{u}\right)=D_{f} \nabla^{2} c_{f}$

$\rho_{f} C_{p, f}\left[\frac{\partial T_{f}}{\partial t}+\nabla \cdot\left(T_{f} \boldsymbol{u}\right)\right]=\lambda_{f} \nabla^{2} T_{f}$

where $\rho_{f}$ and $\mu_{f}$ are the fluid density and the fluid viscosity respectively, $D_{f}$ is the species mass diffusivity in the fluid, and $C_{p . f}$ and $\lambda_{f}$ are the heat capacity and the thermal conductivity of the fluid phase respectively. 
Since in the current work the particles are stationary and the reaction occurs at the particle surface, the only governing equation of the solid phase is the particle thermal energy equation. It calculates the particle temperature based on the fluid-solid heat transfer rate (first term on the right-hand side) and the rate of reaction heat liberated from the catalytic reaction (second term on the righthand side):

$V_{s} C_{p, s} \frac{d T_{s}}{d t}=\Phi_{t h . f \rightarrow s}+\Phi_{s p . f \rightarrow s}\left(-\Delta \mathrm{H}_{\mathrm{r}}\right)$

In this equation, $V_{s}$ is the particle volume, $C_{p, s}$ is the volumetric heat capacity of the particle and $\Delta H_{r}$ is the reaction enthalpy. The heat transfer rate and the mass transfer rate, with the normal pointing inward to the solid, are calculated by integrating of the temperature and concentration gradient at the sphere surface over the whole sphere $\left(S_{s}\right.$ the external surface area of the sphere) using the following two equations respectively:

$\Phi_{t h, f \rightarrow S}=-\iint_{S_{S}}\left(\lambda_{f} \nabla T_{f} \cdot \mathbf{n}\right) d S$

$\Phi_{s p . f \rightarrow s}=-\iint_{S_{s}}\left(D_{f} \nabla c_{f} \cdot \mathbf{n}\right) d S$

Providing an exothermic catalytic reaction and a small enough Biot number, the heat liberation is promptly transported to the interior of the particle with a negligible intra-particle temperature gradient, i.e., a uniform solid temperature over the whole particle.

\subsection{Boundary conditions at the particle surface}

The interfacial boundary condition for the concentration computation is taken such that a first-order irreversible chemical reaction proceeds on the external surface of catalyst particles. There is no accumulation on the particle surface, indicating an equilibrium between the rate of consumption due to reaction and the diffusion flux to the particle surface.

$k c_{f, s}=-D_{f} \frac{\partial c_{f, s}}{\partial \mathbf{n}}$

In this equation, $k$ is the surface reaction rate coefficient with the unit meter per second, $D_{f}$ is the species mass diffusivity and $\mathbf{n}$ is the normal pointing into the sphere. Due to the significant heat effect, the fluid phase thermal energy equation is solved to determine local fluid temperatures. For this purpose, the Dirichlet boundary condition with the particle temperature computed by Equation (6) is enforced at the fluid-solid interface for individual catalyst particles.

The particle temperature rises due to the exothermic catalytic reaction proceeding at the particle surface. Simultaneously, the increased particle temperature results in a higher reaction rate and consequently a higher heat production rate at an increased interfacial mass transfer rate. At steady state, a constant particle temperature is obtained. This implies the liberated reaction heat is fully carried away by the fluid flow. The influence of the particle temperature on the reaction rate coefficient is computed by the well-known Arrhenius equation:

$k=A e^{-\frac{E_{a}}{R T_{s}}}$

where $A$ is the pre-exponential factor, $E_{a}$ is the activation energy of the current catalytic reaction, $R$ is the universal gas constant and $T_{S}$ is the particle temperature in Kelvins. At the end of every time step, the solid temperature and subsequently the reaction rate coefficient of individual particles are computed. They serve as the updated boundary conditions for the fluid phase thermal energy and species equation at the new time step respectively.

It should be noted that the parameters of the Arrhenius equation, namely the pre-exponential factor and the activation energy, as well as the parameters of the system, namely initial temperature and the reaction enthalpy, are adopted from realistic parameters of the methane partial oxidation reaction (POX) (Melchiori et al., 2014) and applied to all simulations in this paper. One should be aware that a single species together with realistic parameters are considered in this paper to demonstrate the applicability of our method for highly coupled catalytic reactions, whereas the realistic POX reaction involves more reactions, namely full combustion, steam reforming, dry reforming and water-gas shifting. Besides that, solid phase reactions should also be well addressed in the modeling of industrial processes. We refer the interested reader to the work of Groote and Froment (1996) for more detailed kinetics.

\subsection{Dimensionless numbers used in the work}

Besides the Biot number given in Equation (1), some more dimensionless numbers used in the current paper are summarized in this sub-section.

Equation (9) that relates the species flux to a particle to the surface reaction can be non-dimensionalized to obtain the definition of the Damköhler number Da using spherical coordinates, namely:

$k c_{f, s}=-D_{f} \frac{\partial c_{f, s}}{\partial r}$

The Damköhler number describes the ratio of the reaction rate to the mass transfer rate:

$D a=\frac{k r_{s}}{D_{f}}$

where $r_{s}$ is the particle radius. A varying reaction rate is characterized by the Damköhler number changing from zero to infinity, indicating a process limited by reaction and mass transfer respectively. It should be noted that the Damköhler number calculated in Equation (12) is the second Damköhler number and is only valid for first order reactions discussed in the current paper.

For fluid-particle systems with only unsteady diffusion and conduction, the Fourier number Fo is computed to be the dimensionless time:

Fo $=\frac{D_{f}}{r_{s}^{2}} t$

where $t$ is the real wall time. For fluid-particle systems with convective transport, the flow behavior is indicated by the particle Reynolds number $R e_{s}$ :

$\operatorname{Re}_{s}=\frac{\rho_{f} u_{0} d_{s}}{\mu_{f}}$

where $u_{0}$ is the superficial fluid velocity and $d_{s}$ is the particle diameter.

The particle-based Sherwood number describes the ratio of the convective mass transfer rate to the diffusive mass transfer rate, whereas the particle-based Nusselt number represents the ratio of the convective heat transfer rate to the conductive heat transfer rate. They are calculated by the following two equations, respectively:

$S h_{s}=\frac{k_{m} d_{s}}{D_{f}}$

$N u_{s}=\frac{\alpha_{h} d_{s}}{\lambda_{f}}$

where $k_{m}$ is the convective mass transfer coefficient and $\alpha_{h}$ is the convective heat transfer coefficient. 
The Schmidt number $S c$ and the Prandtl numberPr, defined as the ratio of the momentum diffusivity to the mass diffusivity and the thermal diffusivity respectively, are given as:

Sc $=\frac{\mu_{f}}{\rho_{f} D_{f}}$

$\operatorname{Pr}=\frac{\mu_{f} C_{p f f}}{\lambda_{f}}$

The Lewis number Le is the ratio of the thermal diffusivity to the mass diffusivity:

$L e=\frac{\frac{\lambda_{f}}{\rho_{f} C_{p, f}}}{D_{f}}=\frac{S c}{\operatorname{Pr}}$

\section{Numerical details}

Equation (2-5) are solved on a staggered Cartesian grid with uniform grid spacing in all three directions, using a finite difference scheme. For temporal discretization, the Adams-Bashforth scheme is used for the convective term and the Euler backward scheme is used for the diffusive term. For spatial discretization, the convection term is discretized by a second-order total variation diminishing scheme and the diffusion term is discretized by a standard second-order central differencing scheme. The momentum equation is advanced through a two-step projection method. The solid phase equation is solved after the fluid phase equations. Following the previous work in our group (Deen and Kuipers, 2014), the particle thermal energy equation is temporally integrated using the following expression:

$T_{s}^{n+1}=T_{s}^{n}+\frac{\Delta t}{V_{s} C_{p, s}}\left[\frac{\Phi_{t h . f \rightarrow s}^{n}+\Phi_{t h, f \rightarrow s}^{n+1}}{2}+\left(-\Delta H_{r}\right) \frac{\Phi_{s p . f \rightarrow s}^{n}+\Phi_{s p . f \rightarrow s}^{n+1}}{2}\right]$

In this equation, the second order trapezoidal rule is applied. The calculation procedure from time step $n$ to $n+1$ is shown in Fig. 1 . The momentum, species and thermal energy equations are solved sequentially, and the stiff coupling between heat and mass transfer is achieved by using a small enough time step.

The discretization of all governing equations leads to generic algebraic equations written as: $a_{c} \phi_{c}+\sum_{1}^{6} a_{n b} \phi_{n b}=b_{c}$

where $\phi$ is the target fluid variable, namely velocity, concentration and temperature fields for Navier-Stokes, species and thermal energy equations, respectively. This equation provides the relationship between any fluid quantity $\phi_{c}$ in the simulation domain and its six neighbors indicated as $\phi_{n b}$. Due to the fact that the immersed object boundaries do not conform to the mesh boundaries, a special treatment is required to enforce the boundary condition at the fluid-solid interface. The ghost points, defined as points inside the solid phase but possessing at least one neighbor in the fluid phase, are used to obtain a solution of the fluid phase governing equations. The fluid-solid coupling in our DNS model is briefly summarized here, and the interested reader may refer to our previous study (Lu et al., 2018a) for a more extensive description.

A second order polynomial is used to approximate a generic variable $\phi$ in the vicinity of the immersed object surface:

$\phi=\sum_{i=0}^{2} \sum_{j=0}^{2} \sum_{k=0}^{2} c_{i j k} x^{i} y^{j} z^{k}, i+j+k \leqslant 2$

It should be noted that relative coordinates with respect to the boundary point $\phi_{B}$ is applied here, and hence this equation can be in fact written as the Taylor expansion:

$$
\begin{aligned}
\phi(x, y, z)= & \phi_{B}+\frac{\partial \phi_{B}}{\partial x} x+\frac{\partial \phi_{B}}{\partial y} y+\frac{\partial \phi_{B}}{\partial z} z+\frac{1}{2} \frac{\partial^{2} \phi_{B}}{\partial x^{2}} x^{2} \\
& +\frac{1}{2} \frac{\partial^{2} \phi_{B}}{\partial y^{2}} y^{2}+\frac{1}{2} \frac{\partial^{2} \phi_{B}}{\partial z^{2}} z^{2}+\cdots
\end{aligned}
$$

The first coefficient is the Dirichlet value, while the second, third and fourth coefficients are the derivative in $\mathrm{x}, \mathrm{y}$ and $\mathrm{z}$ directions respectively. With these, one can enforce the Robin boundary condition at the immersed object surface:

$\alpha \phi_{B}+\beta \frac{\partial \phi_{B}}{\partial \mathbf{n}}=f$

The coefficients are obtained through a reconstruction procedure consisting of nine neighboring fluid points and one image point, written as:

$\phi=X c$

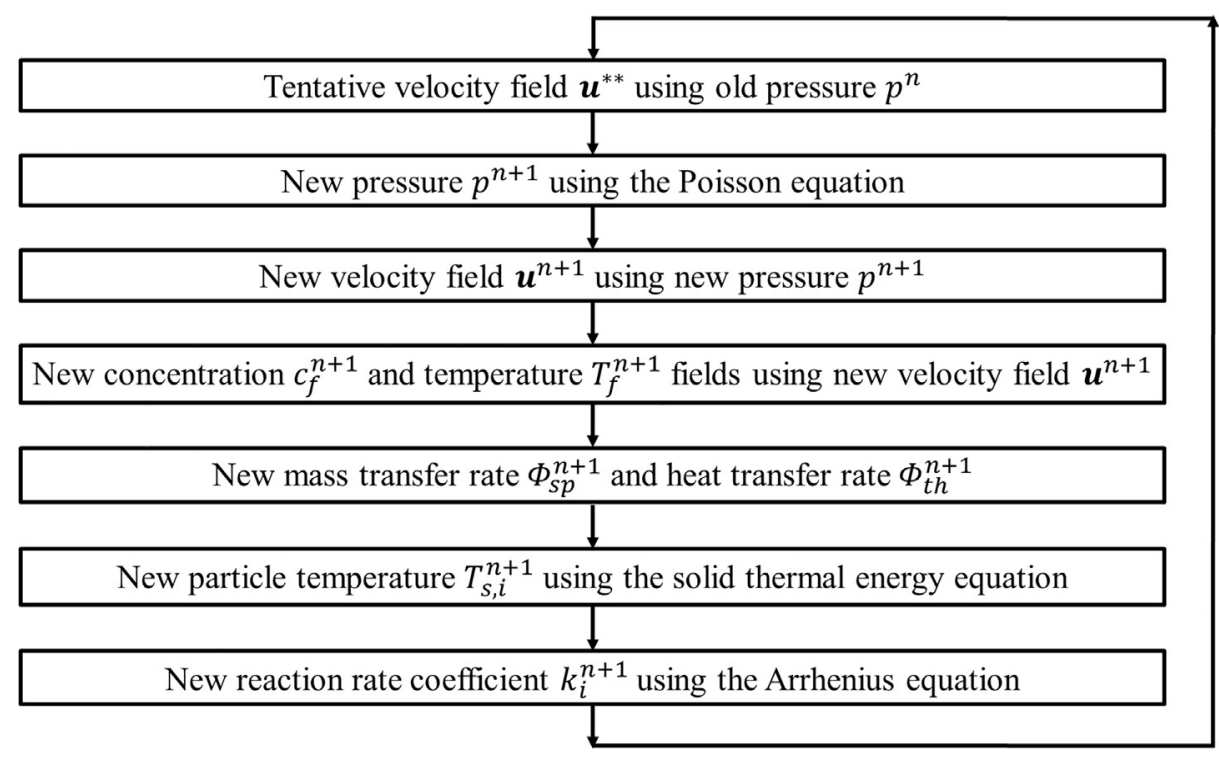

Fig. 1. Flow chart of the simulation over one time step from $t^{n}$ to $t^{n+1}$ 
In this equation, $\boldsymbol{X}$ is the Vandermonde matrix and vectors $\boldsymbol{\phi}$ and $\boldsymbol{c}$ are for fluid variable and polynomial coefficients respectively. The coefficients are expressed as a linear combination of variable values and the relative coordinates. The image point value is first evaluated by satisfying the boundary condition, and subsequently the ghost point value is computed and used to update the matrix coefficients in Equation (21).

\section{Results and discussion}

In this section, results obtained for different fluid-particle systems with an increasing number of particles are presented: a single sphere, three spheres and a dense array. These systems are highly related to engineering applications and demonstrate the strengths of our DNS methodology for modeling of reactive fluid-particle systems.

\subsection{Single sphere system}

In this part, we consider a single stationary sphere positioned in the fluid. The free-slip boundary condition is applied at the domain boundaries for the computation of the velocity field, whereas a homogeneous Neumann boundary is applied there for the species and thermal energy conservation equations indicating an isolated and adiabatic system. At the outlet the pressure is prescribed as the standard atmospheric pressure, and both concentration and temperature boundary conditions are specified as a zero normal gradient. Fluid flows into the system with a prescribed uniform velocity of $0.08 \mathrm{~m} / \mathrm{s}, 0.24 \mathrm{~m} / \mathrm{s}, 0.4 \mathrm{~m} / \mathrm{s}, 0.8 \mathrm{~m} / \mathrm{s}$ and $1.2 \mathrm{~m} / \mathrm{s}$, corresponding to a particle-based Reynolds number of 20, 60, 100, 200 and 300, respectively. The fluid inlet concentration and temperature are set to be the same as the initial values of the system. A mesh resolution $N=20$, defined as the ratio of the sphere diameter to the grid size, has been found to be sufficient in our earlier paper (Lu et al., 2018a). The data used for the numerical simulation are given in Table 1.

At steady state the particle temperature becomes constant, and Equation (6) reduces to:

$\Phi_{s p . f \rightarrow s}\left(-\Delta H_{r}\right)=-\Phi_{t h, f \rightarrow s}$

indicating that the heat liberated from the chemical reaction is fully transferred to the fluid. This equation can be rewritten with the mass transfer coefficient $k_{m}$ and the heat transfer coefficient $\alpha_{h}$ :

$k_{m}\left(c_{f, i n}-c_{f, s}\right)\left(-\Delta H_{r}\right)=-\alpha_{h}\left(T_{f, i n}-T_{s}\right)$

It should be noted that the external surface area of the particle $S_{s}$ is required for both heat and mass transfer calculations and hence

Table 1

Data used for the simulation of the single sphere system.

\begin{tabular}{lll}
\hline Parameter & Value & Unit \\
\hline Particle radius $d_{s}$ & 0.0025 & $\mathrm{~m}$ \\
Fluid density $\rho_{f}$ & 1.0 & $\mathrm{~kg} / \mathrm{m}^{3}$ \\
Fluid viscosity $\mu_{f}$ & $2 \times 10^{-5}$ & $\mathrm{~kg} / \mathrm{m} / \mathrm{s}$ \\
Fluid diffusivity $D_{f}$ & $2 \times 10^{-5}$ & $\mathrm{~m} / \mathrm{s}$ \\
Fluid thermal conductivity $\lambda_{f}$ & 0.025 & $\mathrm{~W} / \mathrm{m} / \mathrm{K}$ \\
Fluid heat capacity $C_{p, f}$ & 1000 & $\mathrm{~J} / \mathrm{kg} / \mathrm{K}$ \\
Particle volumetric heat capacity $C_{p, s}$ & 1000 & $\mathrm{~J} / \mathrm{m}^{3} / \mathrm{K}$ \\
Reaction enthalpy $\Delta H_{r}$ & $-3 \times 10^{4}$ & $\mathrm{~J} / \mathrm{mol}$ \\
Fluid initial concentration $c_{f, 0}$ & 10 & $\mathrm{~mol} / \mathrm{m}^{3}$ \\
Fluid initial temperature $T_{f, 0}$ & 900 & $\mathrm{~K}$ \\
Particle initial temperature $T_{s, 0}$ & 900 & $\mathrm{~K}$ \\
Pre-exponential factor $A$ & $10^{5}$ & $\mathrm{~m} / \mathrm{s}$ \\
Activation energy $E_{a}$ & $10^{5}$ & $\mathrm{~J} / \mathrm{mol}$ \\
\hline
\end{tabular}

cancelled. By means of equating the external mass flux to the particle surface and the reaction consumption, one obtains:

$k_{m}\left(c_{f, i n}-c_{f, s}\right)=k c_{f, s}$

With Equation $(12,27,28)$, the particle temperature at steady state is obtained, given by the following equation:

$T_{s}=T_{f, i n}+\frac{2 D a}{\left(2 D a+S h_{s}\right)} \frac{S h_{s}}{N u_{s}} \frac{c_{f, i n}\left(-\Delta H_{r}\right)}{\rho_{f} C_{p, f} L e}$

Note that the Damköhler number is temperature dependent due to the Arrhenius dependence of the reaction rate, so Equation (29) is in fact a non-linear equation with respect to $T_{s}$.

A special case without convective transport is first considered. For this circumstance, the transport phenomena for unsteady species diffusion and heat conduction in the fluid phase are also described by Equation (4) and (5) respectively, but setting $\boldsymbol{u}$ to zero. In DNS, the boundary conditions for both species and thermal energy equations at the simulation domain are fixed as the initial conditions. They are valid as long as the fronts of unsteady diffusion and conduction have not reached the simulation boundaries. For efficient utilization of the computational resource, the particle is located in the center of a cubic box with 360 grid cells in each direction. To offer an "exact" solution for the evolution profile of the particle temperature, the 1D radial model written in spherical coordinate is solved by a standard second order finite difference technique. It should be noted that all angular derivatives are zero due to symmetry. In this case, the governing equations for unsteady transport phenomena in the fluid phase are respectively described as:

$\frac{\partial c_{f}}{\partial t}=\frac{D_{f}}{r^{2}} \frac{\partial}{\partial r}\left(r^{2} \frac{\partial c_{f}}{\partial r}\right)$

$\rho_{f} C_{p, f} \frac{\partial T_{f}}{\partial t}=\frac{\lambda_{f}}{r^{2}} \frac{\partial}{\partial r}\left(r^{2} \frac{\partial T_{f}}{\partial r}\right)$

The heat and mass transfer rates required in the solid phase thermal energy equation are redefined as:

$\Phi_{t h . f \rightarrow s}=-\left.\lambda_{f} \frac{\partial T_{f}}{\partial r}\right|_{r=R_{s}} 4 \pi R_{s}^{2}$
$\Phi_{s p . f \rightarrow s}=-\left.D_{f} \frac{\partial c_{f}}{\partial r}\right|_{r=R_{s}} 4 \pi R_{s}^{2}$

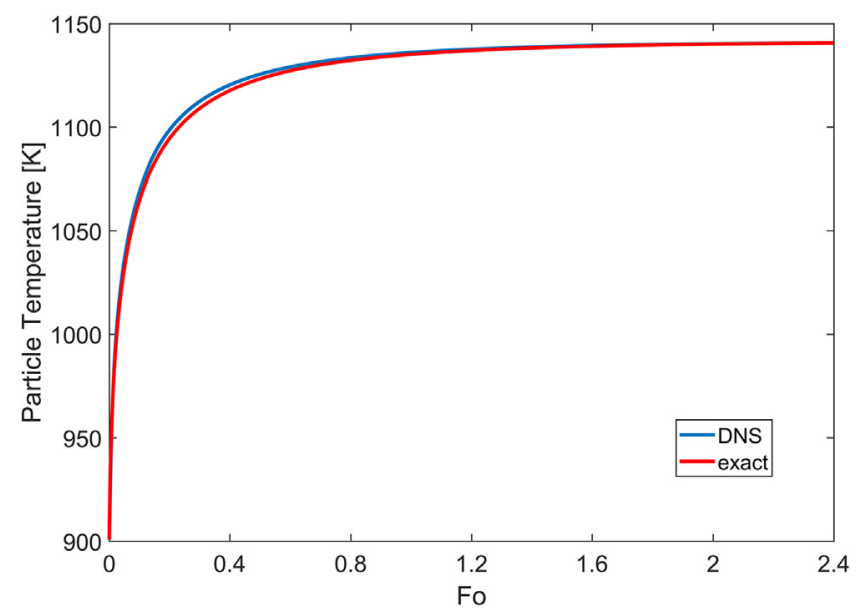

Fig. 2. Comparison of the evolution profile of the particle temperature between the DNS result and the "exact" solution, for the limiting case of unsteady mass and heat diffusion around a single stationary sphere. 
It should be noted here that across the particle diameter a large number of computational cells was used to obtain this highly accurate solution. In Fig. 2 the particle temperature evolution profile is plotted as a function of the dimensionless time Fo, to give a comparison between the DNS result and the "exact" solution. As clearly demonstrated in the figure, these two solutions reach a good agreement. The slight difference might originate from different time integration methods, as a second order one is used in the "exact" solution. The species flux is relatively high at initial time steps, so that the heat liberated from the exothermic reaction sharply heats the particle up. Consequently, the reaction rate is further increased and the heat is transferred from the particle to the surrounding fluid through unsteady heat conduction. The particle temperature attains a constant value as ultimately the heat removal rate equals the heat liberation rate.

For the simulations with convective mass and heat transfer, the sphere is located at the center of the domain laterally while it is positioned at a distance of two times of the sphere size from the inlet in the flow direction. From Equation (29), the final particle temperature can be iteratively computed, using the Sherwood number and the Nusselt number provided by the well-known empirical Frössling and Ranz-Marshall correlations respectively:

$S h_{s}=2.0+0.6\left(R e_{S}\right)^{\frac{1}{2}}(S c)^{\frac{1}{3}}$

$N u_{s}=2.0+0.6\left(R e_{s}\right)^{\frac{1}{2}}(\operatorname{Pr})^{\frac{1}{3}}$

In Table 2, the comparison is shown for the particle temperature at steady state between the DNS results and the empirical values. An interesting discrepancy is that, compared to the empirical results, the DNS gives particle temperatures of a few degrees higher at low Reynolds numbers and few degrees lower at high Reynolds numbers. To facilitate the investigation of the cause, the Sherwood number and the Nusselt number obtained from the DNS are also listed in the table. They are computed by the following two equations respectively:

$$
\begin{aligned}
& S h_{s}=\frac{\Phi_{s p, f \rightarrow s}}{4 \pi R_{s}^{2}\left(c_{f, s}-c_{f, i n}\right)} \frac{d_{s}}{D_{f}} \\
& N u_{s}=\frac{\Phi_{t h, f \rightarrow s}}{4 \pi R_{s}^{2}\left(T_{s}-T_{f, i n}\right)} \frac{d_{s}}{\lambda_{f}}
\end{aligned}
$$

where the mass transfer rate $\Phi_{s p . f \rightarrow s}$ and the heat transfer rate $\Phi_{t h . f \rightarrow s}$ are the same ones used in the solid phase thermal energy equation. As predicted by the empirical correlations, the interfacial mass and heat transfer performance are both enhanced with increasing Reynolds numbers, and the Sherwood numbers are always larger than the Nusselt numbers due to the slightly thinner mass boundary layer ( $S c=1$ vs. $P r=0.8$ ). However, different deviations are noticed for the Sherwood number and the Nusselt number at the same Reynolds number, and this small difference leads to the discrepancy of the final particle temperature. In other words, DNS simulation is quite sensitive to the relative deviation between the Sherwood number and the Nusselt number. For example, at $R e_{s}=20$ the heat transfer rate is slightly lower and hence the particle temperature is higher than the empirical value, while at $R e_{s}=300$ the mass transfer rate is slightly lower and hence the particle temperature is lower than the empirical value.

It is expected that the Sherwood and Nusselt numbers obtained from DNS are smaller than the empirical values, as this underestimation has already been reported by other researchers (Feng and Michaelides, 2000; Richter and Nikrityuk, 2012; Kravets and Kruggel-Emden, 2017). The slightly different deviations for the heat and mass transfer processes can be reasonably explained by the different boundary conditions. To be specific, $c_{f, s}$ in Equation (36) is the average species concentration of local values over the sphere surface, while $T_{s}$ in Equation (37) is constant everywhere on the sphere surface.

The heat and mass balance of our DNS model can be verified by computing the theoretical values of the particle temperature using the Sherwood, Nusselt and Damköhler numbers obtained from the DNS results. The comparison is listed in Table 2 and shows good agreement. One can further observe that the final particle temperatures are not sensitive to the change of the particle Reynolds numbers. It can be explained as follows: in case the Damköhler number is much larger than the particle Sherwood number and the convection term dominates, the reaction-transport competition term becomes unity and the heat-mass coupling term is simplified, respectively. The effective reaction rate is nearly fully transport limited. Under these circumstances, Equation (29) is rewritten as the following expression:

$T_{s}=T_{f, i n}+\frac{(S c)^{\frac{1}{3}}}{(P r)^{\frac{1}{3}}} \frac{c_{f, i n}\left(-\Delta H_{r}\right)}{\rho_{f} C_{p, f} L e}=T_{f, i n}+\frac{c_{f, i n}\left(-\Delta H_{r}\right)}{\rho_{f} C_{p, f} L e^{\frac{2}{3}}}$

and one can clearly observe its independence on the particle Reynolds number.

\subsection{Three spheres system}

In this part, the three-bead reactor is considered. In this reactor, three catalytic particles are aligned in a queue. The spheres are positioned in a square duct that is $0.08 \mathrm{~m}$ in length and $0.01 \mathrm{~m}$ in width. At the inlet fluid flows into the system with a uniform velocity and constant concentration and temperature, while at the outlet the pressure is prescribed as the standard atmospheric pressure and for both concentration and temperature the zero gradient boundary condition is imposed. At the lateral boundaries of the simulation domain, free slip boundary conditions are applied for the velocity field calculation and zero-flux Neumann boundary conditions are applied for the concentration and temperature field calculation. In the streamwise direction, the first sphere is located at a distance of two times of the sphere diameter from the inlet, and the other two spheres are located in such a way that the mutual distance between all sphere centers is one and half times of the sphere diameter. For the simulation three particle-based Reynolds numbers of 60,120 and 240 are investigated, and all

Table 2

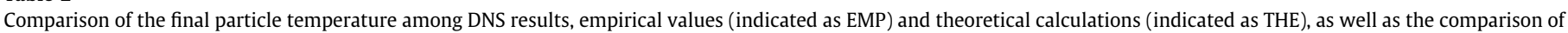

\begin{tabular}{|c|c|c|c|c|c|c|c|c|c|}
\hline \multirow[t]{2}{*}{$R e_{s}$} & \multicolumn{3}{|l|}{$\underline{T_{s}}$} & \multicolumn{3}{|l|}{$S h_{s}$} & \multicolumn{3}{|l|}{$N u_{s}$} \\
\hline & DNS & EMP & THE & DNS & EMP & DEV & DNS & EMP & DEV \\
\hline 20 & 1150.1 & 1148.6 & 1149.8 & 4.42 & 4.68 & $-5.5 \%$ & 4.22 & 4.49 & $-6.0 \%$ \\
\hline 60 & 1150.4 & 1150.3 & 1150.5 & 6.38 & 6.65 & $-4.1 \%$ & 6.06 & 6.31 & $-4.0 \%$ \\
\hline 100 & 1149.9 & 1150.8 & 1150.1 & 7.70 & 8.00 & $-3.7 \%$ & 7.31 & 7.57 & $-3.4 \%$ \\
\hline 200 & 1147.9 & 1151.1 & 1147.9 & 10.12 & 10.49 & $-3.5 \%$ & 9.66 & 9.88 & $-2.2 \%$ \\
\hline 300 & 1145.2 & 1151.0 & 1145.1 & 11.63 & 12.39 & $-6.1 \%$ & 11.20 & 11.65 & $-3.9 \%$ \\
\hline
\end{tabular}

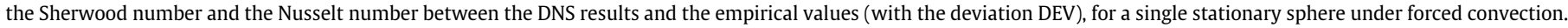


other simulation parameters are the same as those in the previous single sphere system.

Fig. 3 illustrates the simulation results of the concentration and temperature distributions in the central plane of the three-bead reactor at steady state for all three Reynolds numbers. It should be noted that inside the particle the concentration is zero whereas the temperature is uniform. At such high temperature, the catalytic reaction proceeds fast and hence the concentration at the particle surface $c_{f, s}$ is almost zero. In the figure, the influence of the fluid velocity is clearly visible in the wake behind the spheres. In the wake behind the third sphere, the concentration (temperature) field develops from a central region to two circulating eddy rings. The final particle temperatures together with the corresponding Sherwood numbers and Nusselt numbers are listed in Table 3. The Sherwood and Nusselt numbers are computed by Equation (36) and (37) respectively. As expected the interfacial transfer performance is improved at higher Reynolds numbers. It is observed that both heat and mass transfer have a worse performance for the downstream spheres. This is due to the shielding effect of the upstream particles. The particle temperature at steady state is of high interest, as both convective heat transfer and liberated reaction heat contribute to the rise of the particle temperature. The upstream particle transfers the thermal energy to the fluid and subsequently to downstream particles, simultaneously unconverted species reacts to give additional heating. At the particle Reynolds number of 60 , three particles have solid temperatures that only differ slightly in the steady state. The slightly lower value of the $2^{\text {nd }}$ sphere is understood as a blockage effect, whereas the small increase of the $3^{\text {rd }}$ sphere temperature may result from the fully developed fluid flow behind the sphere. At the particle Reynolds number of 120 , similar behavior is observed with even closer values of the particle temperatures. At the particle Reynolds number of 240 , the system has a completely different behavior that the particle temperature increases from the $1^{\text {st }}$ sphere to the $3^{\text {rd }}$ sphere. In this circumstance, the convective heat transfer plays an important role because the thermal energy transferred from the fluid to the sphere leads to a further increase of the particle temperature and consequently more reaction heat liberated at an improved reaction rate.

The axial profiles of cup-average concentration and temperature in the fluid phase are shown in Fig. 4. In this figure, the relative contribution of individual spheres to the overall reactant conversion and temperature rise is demonstrated. The cup-average value is calculated by the following equation:

$\left\langle\phi_{f}\right\rangle=\frac{\iint_{S_{f}} u_{x}(x, y, z) \phi_{f}(x, y, z) d y d z}{\iint_{S_{f}} u_{x}(x, y, z) d y d z}$

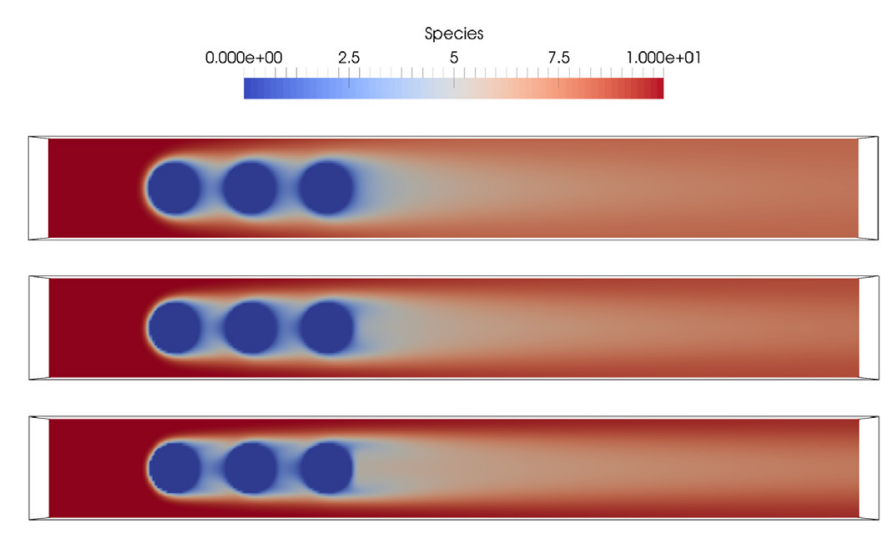

Table 3

Solid temperature, Sherwood number and Nusselt number of each sphere at steady state, for three particle Reynolds numbers.

\begin{tabular}{lllll}
\hline$R e_{s}$ & & 60 & 120 & 240 \\
\hline $1^{\text {st }}$ sphere & $T_{s}$ & 1151.3 & 1150.5 & 1142.2 \\
& $S h_{s}$ & 6.78 & 8.71 & 10.89 \\
\multirow{2}{*}{$2^{\text {nd }}$ sphere } & $N u_{s}$ & 6.42 & 8.24 & 10.61 \\
& $T_{s}$ & 1150.8 & 1150.2 & 1144.9 \\
& $S h_{s}$ & 4.09 & 5.02 & 6.18 \\
$3^{\text {rd }}$ sphere & $N u_{s}$ & 3.89 & 4.78 & 6.00 \\
& $T_{s}$ & 1151.1 & 1150.4 & 1147.8 \\
& $S h_{s}$ & 3.61 & 4.44 & 5.75 \\
& $N u_{s}$ & 3.44 & 4.23 & 5.52 \\
\hline
\end{tabular}

In this equation, the integration of the fluid variable $\phi_{f}$ (concentration $c_{f}$ or temperature $T_{f}$ ) is performed over a cross-sectional surface $S_{f}$ (with only the part occupied by the fluid) perpendicular to the flow direction $x$. For all Reynolds numbers, the first sphere has the largest contribution. The second sphere has a lower contribution compared with the first sphere, whereas the third sphere contributes even less. This observation agrees well with the particle Sherwood numbers and Nusselt numbers listed in the last table. With increasing Reynolds numbers the reactant conversion at all sphere surfaces decreases. This results in a lower particle temperature and consequently less thermal energy is transferred from the solid phase to the fluid phase.

From the simulation, the species overall conversion ratio and the fluid temperature rise are calculated based on the difference of the cup-average concentration and temperature between the inlet and the outlet:

$$
\xi_{f}=\frac{\left\langle c_{f, \text { in }}\right\rangle-\left\langle c_{f, \text { out }}\right\rangle}{\left\langle c_{f, \text { in }}\right\rangle}
$$

$$
\Delta T_{a}=\left\langle T_{f, o u t}\right\rangle-\left\langle T_{f, \text { in }}\right\rangle
$$

For the particle Reynolds numbers of 60,120 and 240, the species overall conversion ratio is $0.194,0.125$ and 0.085 respectively and the adiabatic temperature rise is $57.4 \mathrm{~K}, 36.6 \mathrm{~K}$ and $24.1 \mathrm{~K}$ respectively. Theoretically, the adiabatic temperature rise can be predicted by the species overall conversion ratio using the following equation:

$$
\Delta T_{a}=\frac{\left(-\Delta H_{r}\right) C_{f, i n} \xi_{f}}{\rho_{f} C_{p . f}}
$$

Using this equation, the adiabatic temperature rise is computed to be $58.2 \mathrm{~K}, 37.5 \mathrm{~K}$ and $25.5 \mathrm{~K}$ for increasing particle Reynolds
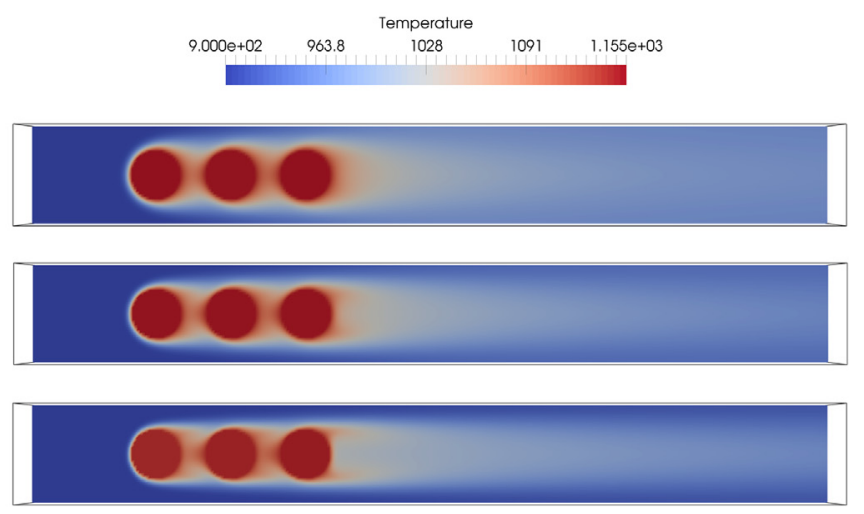

Fig. 3. Species concentration (left) and temperature (right) distribution of the central plane inside the reactor. From top to bottom, the particle Reynolds number is 60 , 120 and 240 , respectively. 


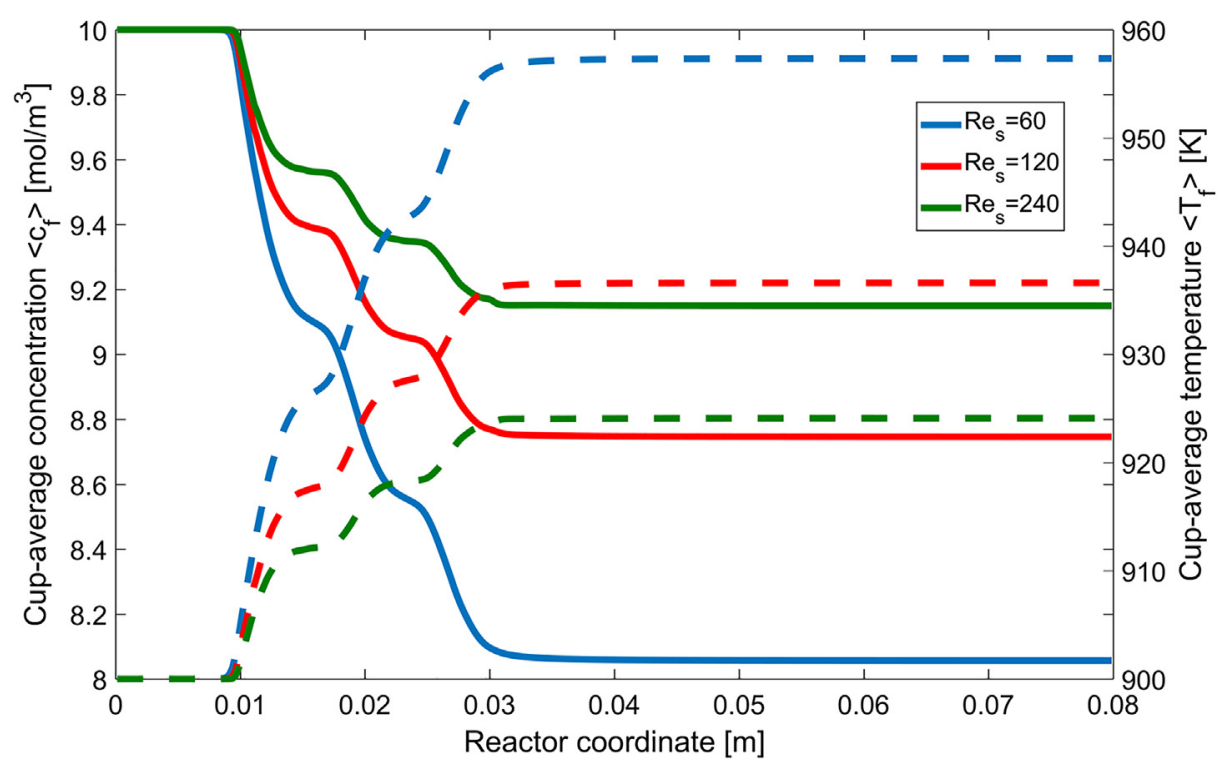

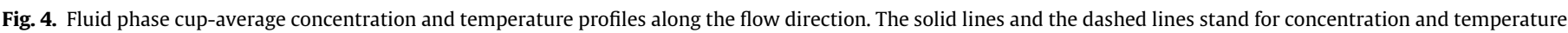

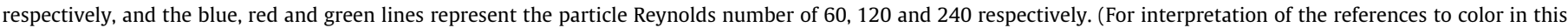
figure legend, the reader is referred to the web version of this article.)

numbers, showing good agreement with the values obtained from DNS results.

\subsection{Dense array system}

As the last simulation case in this paper, we further increase the number of particles to make a more physically complex system, namely a dense stationary array consisting of 645 randomly configured catalytic particles. This particle array is generated by the hard-sphere Monte-Carlo method and distributed with a predefined bed voidage $\varepsilon$ of 0.7 . The 645 particles are of the same size, and periodically repeated in the two lateral directions. In other words, although part of these particles seem to be cut by the domain boundaries, they actually enter the simulation domain again at the opposite boundary. In the simulation, fluid flows through the array and the previously mentioned POX-adopted first order irreversible chemical reaction proceeds at the surface of the particles. The simulations are performed on a 3D domain with a length of $0.165 \mathrm{~m}$ in the flow direction and a length of $0.0375 \mathrm{~m}$ in the lateral directions. The first 60 grid cells and the last 200 grid cells in the flow direction are reserved as inlet and outlet regions respectively, hence all particles are located within the reactor coordinates from $0.015 \mathrm{~m}$ to $0.115 \mathrm{~m}$. The incorporation of these two empty sections is essential to avoid problems for inlet flow development and outflow recirculation respectively, especially at high fluid velocities. At the inlet, a prescribed uniform fluid velocity has the value of $0.32 \mathrm{~m} / \mathrm{s}, 0.64 \mathrm{~m} / \mathrm{s}$ and $0.96 \mathrm{~m} / \mathrm{s}$, corresponding to the particle Reynolds number of 80,160 and 240 , respectively. The fluid enters the system with a uniform concentration of $10 \mathrm{~mol} / \mathrm{m}^{3}$ and a uniform temperature of $900 \mathrm{~K}$. At lateral domain boundaries, periodic boundary conditions are applied for all computations of the velocity, concentration and temperature fields. At the outlet, the pressure is prescribed as the standard atmospheric pressure and both concentration and temperature gradients are set to be zero normal gradient. The same parameters applied in the previous two systems are used for current simulations. For the dense particle array simulations, extra attention needs to be paid to the time step. A value of $1 \times 10^{-6} \mathrm{~s}$ was applied to suppress stability problems related to the stiffness of the nonlinear heat-mass coupling, provided by the Arrhenius equation.
This problem is more pronounced at low Reynolds numbers, as the temperature rise is larger and consequently the increase of the reaction rate coefficient is sharper. The time step can be reset to the CFL-determined value after around $t=1.5 \tau$, where $\tau$ is the residence time of the particle array computed as the ratio of the packing length to the fluid superficial velocity.

The particle configuration is shown in Fig. 5, together with the computed axial velocity distributions at three particle Reynolds numbers over the central plane of the particle array. For the simulations, the ratio of the particle diameter to the grid size is 20 . This value is applied according to the mesh convergence test reported in our earlier publication (Lu et al., 2018a). It should be noted that in the current work we also have the temperature field. The mesh resolution applied is still safe, as the Prandtl number is 0.8 indicating a similar thickness compared with the one of the mass and the momentum boundary layer. In this figure, the periodically distributed particle configuration as well as the periodic velocity field across the domain boundaries are clearly demonstrated. The axial velocity fields are normalized by dividing by the corresponding inlet velocity, and the way that the fluid meanders through the particle array is visualized. Stagnation zones are noticed in front of particles. Preferred flow pathways are observed inside the array, as a pronounced property of the non-homogeneous sphere distribution. In other words, the varying local porosity (less blocked regions) leads to a significant increase of the local fluid velocity. With increasing Reynolds numbers, some streams are intensified while some are abated. Inside the particles, the computed velocity field is zero due to the enforcement of the no-slip boundary condition at the sphere surface. With higher Reynolds numbers, the wake behind the particle array becomes more unstable, and a few circulating vortices are clearly observed at $R e_{s}=240$. As expected, negative values occur at the rear of certain particles. This indicates that local backflow becomes more significant at higher Reynolds numbers.

In Fig. 6 the computed concentration and temperature fields at the time moment of $3.355 \tau$ are shown for all three Reynolds numbers. Taking advantage of our DNS model, the detailed local information in the fluid phase as well as in the solid phase is depicted. The concentration inside the particles is zero due to the particular assumption of a reactive external surface while the temperature 

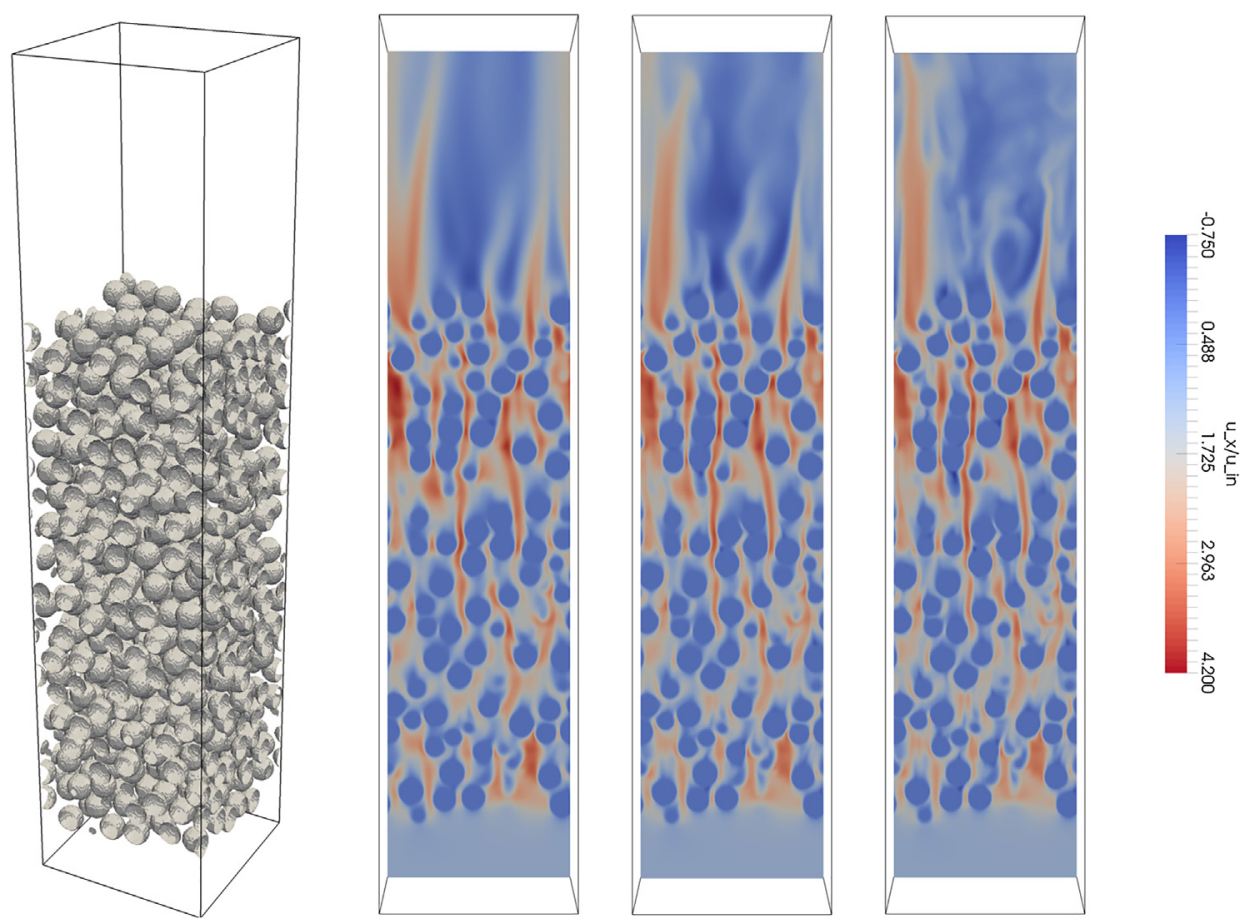

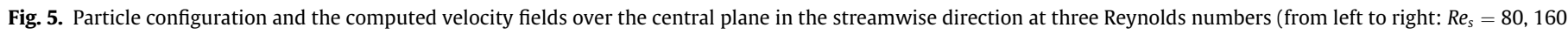
and 240).

inside the particles is the solid temperature computed by Equation (6). For easy comparison, the same color legends are used for the three Reynolds numbers. From the figure, the influence of higher fluid velocities, namely shorter residence times, is apparent because the species conversion is decreased and consequently the temperature rise is lower. In the figure of $R e_{s}=240$, the wake behind individual particle can even be observed at the rear part of the array that the concentration is lower and the temperature is higher than the surrounding fluid, indicating less saturation. To offer a quantitative measurement, the cup-average concentration and temperature are computed. These quantities are of considerable interest for practical applications in industry, as they may help to achieve a more efficient production process such that no noble catalysts are unused and no flow circulation is required. In Fig. 7 the profiles of the cup-average quantities, computed by Equation (39), are shown in the packing range as a function of the axial position non-dimensionalized by the particle diameter. The concentration profiles decay sharply in the front part of the array due to the large driving force for the interfacial mass transfer, although the reaction rates are smaller than the ones in the rear part. Nearly complete species conversion is obtained, and the fluid is heated up by the liberated reaction heat. Given by DNS, the species overall conversion ratios are $0.997,0.985$ and 0.973 , and the system fluid temperature rises are $284.1 \mathrm{~K}, 273.8 \mathrm{~K}$ and $266.0 \mathrm{~K}$, for the particle Reynolds number 80, 160 and 240 respectively. Deviations with the theoretical values computed by Equation (42), specifically $15.0 \mathrm{~K}, 21.7 \mathrm{~K}$ and $25.9 \mathrm{~K}$ for increasing Reynolds numbers, are observed. This discrepancy will be further analyzed and discussed in the following part with transient profiles.

In Fig. 8, the transient behavior of the catalytic reaction process is illustrated over the longitudinal central plane at the particle Reynolds number of 240 . At the initial stage, the exothermic chemical reaction proceeds at the external surface of catalyst particles with the prescribed ignition temperature. Due to the relatively fast reaction rate, it quickly consumes the surrounding species. Heat is liberated to heat up individual particle, and all particles have almost the same temperature. Later on, thermal energy transfers from the heated particles to the fluid flowing around the particles. The fluid temperature in the wake region is considerably increased, while the fluid flowing through the interstitial void spaces is comparatively colder. Subsequently, the temperature of catalysts in the front part of the array keeps increasing due to the large mass transfer driving force, and the unconverted species is transported to downstream particles. At this moment, the solid temperatures in the front part of the array are pronouncedly higher than those in the rear part, and the reactant is not fully consumed by the catalysts. Meanwhile, more heat is carried away by the fluid from front particles and propagates to downstream particles. With a higher temperature, the reaction rate of downstream catalysts rises and more reactant is converted through the surface reaction. This gives additional heating to the particles and accordingly sweeps the energy wave to farther downstream particles. At the final stage, the temperature of catalysts increases in the flow direction. The particles store the liberated reaction energy and the flow transports the thermal energy through the particle array. The reactant gets almost fully converted by the chemical reaction at such a high but different reaction rate depending on individual particles.

In reactor modeling, the one-dimensional heterogeneous model is popular to provide a quick estimation of the heat and mass transport processes inside such a particle array. One reads:

$$
\begin{aligned}
& \varepsilon \frac{\partial c_{f}}{\partial t}+u_{i n} \frac{\partial c_{f}}{\partial x}=-k_{m} a_{s}\left(c_{f}-c_{f, s}\right) \\
& \varepsilon \rho_{f} C_{p . f} \frac{\partial T_{f}}{\partial t}+u_{i n} \rho_{f} C_{p . f} \frac{\partial T_{f}}{\partial x}=-\alpha_{h} a_{s}\left(T_{f}-T_{s}\right)
\end{aligned}
$$

for the fluid phase and

$$
\begin{aligned}
& (1-\varepsilon) \frac{\partial c_{f, s}}{\partial t}=-k_{m} a_{s}\left(c_{f, s}-c_{f}\right)-k a_{s} c_{f, s} \\
& (1-\varepsilon) C_{p, s} \frac{\partial T_{s}}{\partial t}=-\alpha_{h} a_{s}\left(T_{s}-T_{f}\right)+k a_{s} c_{f, s}\left(-\Delta H_{r}\right)
\end{aligned}
$$



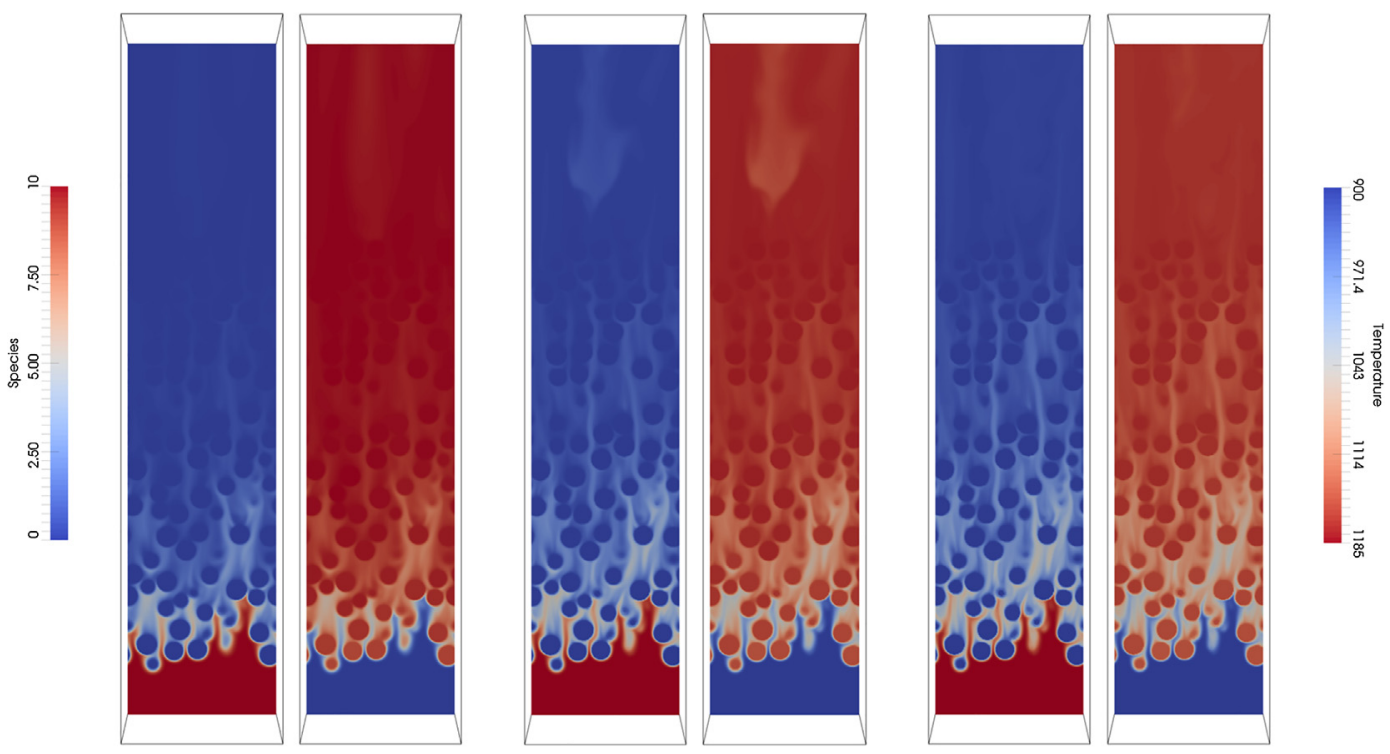

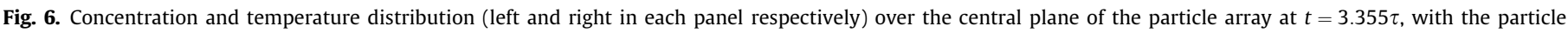
Reynolds number of 80, 160 and 240 from left to right.

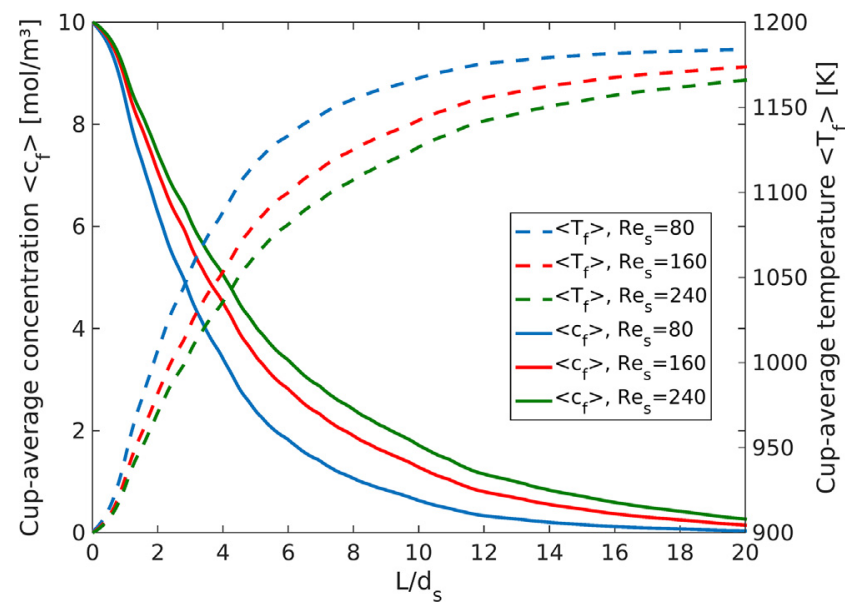

Fig. 7. Cup-average concentration and temperature as a function of the dimensionless axial position for three particle Reynolds numbers at $t=3.355 \tau$. Only profiles for the packing zone are shown. The solid lines and the dashed lines stand for concentration and temperature, and the blue, red and green lines represent the particle Reynolds number of 60,120 and 240 respectively. (For interpretation of the references to color in this figure legend, the reader is referred to the web version of this article.)

for the solid phase. It should be noted that the accumulation term in Equation (45) is actually zero due to our particular assumption of a surface reaction. In above equations, $a_{s}$ is the specific fluid-particle contact surface area

$a_{s}=6 \frac{(1-\varepsilon)}{d_{s}}$

and the mass and heat transfer coefficients are predicted by a widely used correlation from Gunn (1978). Based on a range of experiments, Gunn correlation captures the dependence of porosity, Reynolds number and Schmidt number (replaced by Prandtl number for passive heat transfer processes).

$$
\begin{aligned}
S h_{s}= & \left(7-10 \varepsilon+5 \varepsilon^{2}\right)\left(1+0.7 \operatorname{Re}_{s}^{\frac{1}{5}} S c^{\frac{1}{3}}\right) \\
& +\left(1.33-2.40 \varepsilon+1.20 \varepsilon^{2}\right) \operatorname{Re}_{s}^{\frac{7}{10}} S C^{\frac{1}{3}}
\end{aligned}
$$

It should be noted that axial dispersion is neglected in both fluid mass and heat equations, due to the dominant role of the convective transport for the Reynolds numbers considered in the current work. The unsteady equations (43-46) are solved using a standard finite difference technique implemented in MATLAB. The comparisons between the 1D model and our DNS results for both fluid and solid phase are shown in Fig. 9 and Fig. 10 respectively. The streamwise profiles of both concentration and temperature are compared at five intermediate time steps with the particle Reynolds number of 160 . In both figures, the overall features of the transient profiles agree very well. Even the characteristics at the very early time moment $t=0.335 \tau$ are captured: (1) at the front part of the particle array the profile slightly differs from the profiles at later time steps; (2) at the rear part of the particle array the profile has nearly constant values. Nevertheless, some discrepancies are noticed. The species reacts faster in DNS. This leads to sharper decays and higher conversions in the fluid phase as well as the lower reactant concentration at the particle surface. In DNS, the fluid leaves the particle array with a lower temperature. The difference between the DNS results and the 1D model prediction increases along with the time development, and this originates from the lower solid phase temperature in the DNS. These discrepancies are thought to be caused by two factors: (1) the non-homogeneous particle distributions, as 1D model assumes uniform flow pattern inside the array as well as negligible concentration and temperature changes in the radial direction. The strong variations of the fluid flow in such a dense particle array, causes differences in degree of back mixing and radial dispersion; (2) the IBM itself. As revealed by the single sphere case, the IBM used in the current work is very sensitive to the different deviations between heat and mass transfer rates. Even slight differences will lead to lower particle temperatures by a few degrees. Considering the large number of particles and the even higher interstitial Reynolds number in the dense array, it is expected to induce larger deviations of the solid phase temperature. Taking one step back, this might be a common problem for all non-body conforming methods, as at such a high level of heat-mass coupling every small discretization error in one problem has a significant effect on the other problem and vice versa. This however needs to be further investigated.

In Fig. 6 as well as Fig. 8, it can always be observed that particles at the (nearly) same streamwise coordinate possess different 

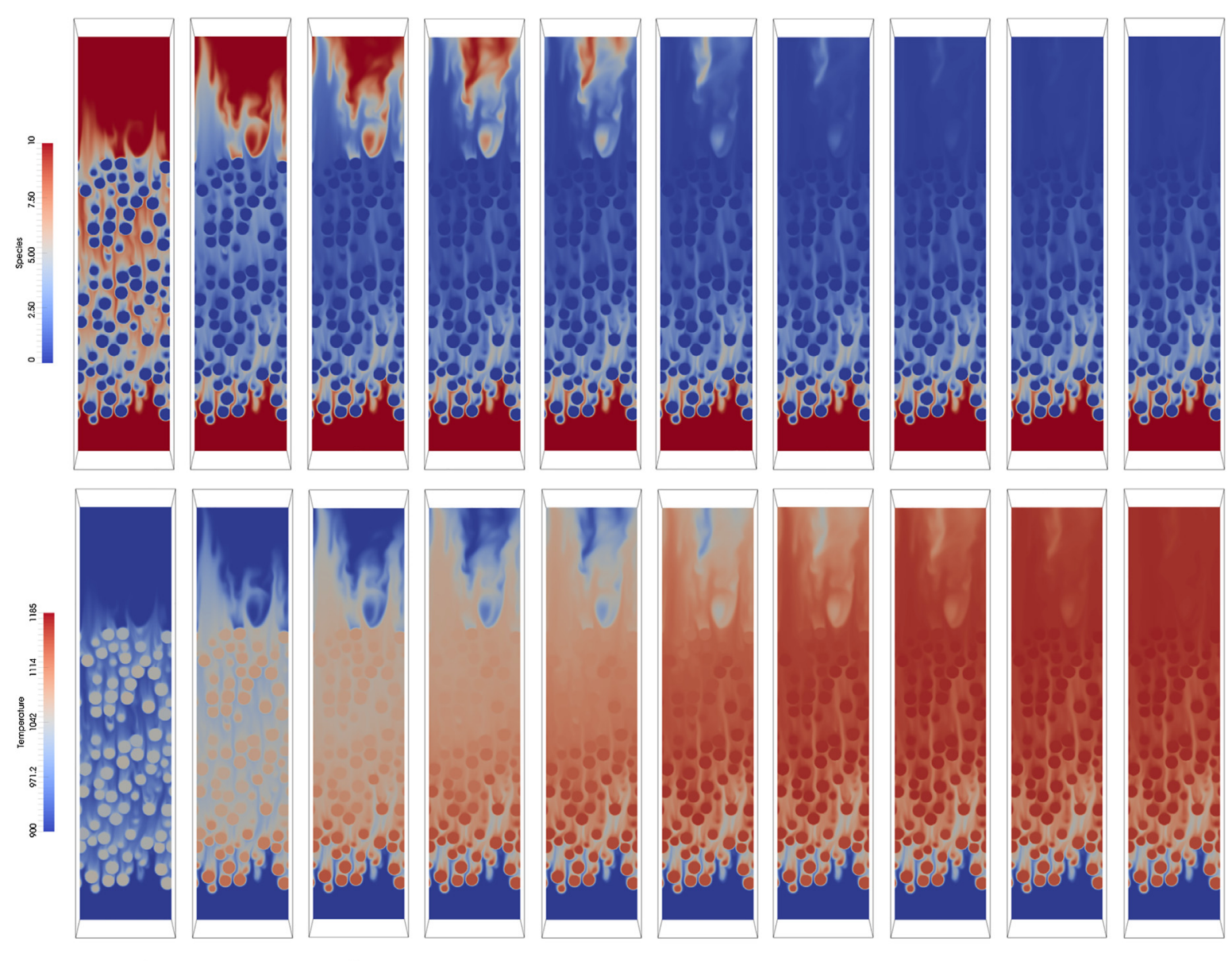

$1.276 \tau$

$1.660 \tau$

$2.140 \tau$

$2.620 \tau$

$3.292 \tau$

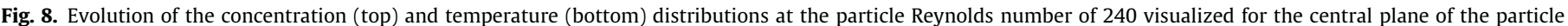
array.

temperatures. In Fig. 11, the solid temperature of individual particles is plotted along the streamwise direction, indicating variations clearly. In the left panel, particle temperatures are plotted at the same time step for three different fluid velocities. For all Reynolds numbers, the variation of the particle temperatures in the transversal cross-sections decreases in the flow direction. This shows a more homogeneous temperature field at the rear part of the particle array. However, the variations increase at higher Reynolds numbers. This confirms the existence of a more heterogeneous flow field inside the particle array. In the right panel, particle temperatures are plotted at three intermediate time steps at the particle Reynolds number of 160 . The variation of particle temperatures significantly decreases with time, even for the very front part of the particle array. In other words, the system tends to reach thermal saturation with a uniform temperature distribution in the radial direction. To obtain a clear visualization, a transversal cross-section at the packing height of $0.055 \mathrm{~m}$ $\left(\frac{L}{d_{s}}=11\right)$ is plotted in Fig. 12 at $t=3.292 \tau$ at the particle Reynolds number of 240 . The temperature of individual particles is shown, and differences up to $4.6 \mathrm{~K}$ can be noted. Compared with the corresponding velocity distribution, it is clearly demonstrated that particles with lower temperature experience local high fluid velocities. In other words, the preferential fluid pathways result in considerable differences in the local fluid-solid transfer rates and as a consequence local regions with varied reaction rate and catalyst temperature. These deviations are due to the presence of all other particles, and the fluid-solid interaction of each individual particle needs to be considered by identifying the configuration of local neighboring particles. However, we failed to correlate the solid temperature with local porosity, after trying to define a local volume using both sphere and cuboid geometries with size varying from $d_{s}$ to $5 d_{s}$. Similar conclusion was given by Akiki et al. (2016), who studied the influence of local parameters on the force on individual particles and suggested to use an anisotropic measure of the neighborhood to obtain a better correlation.

Nevertheless, detailed information regarding individual particles can be obtained from DNS. This may help to understand the heterogeneity better. For this purpose, we calculate the particlebased Sherwood and Nusselt numbers based on a local control volume suggested by Jackson (1997), Link et al. (2005) and Deen et al. (2012). In their work, a cubic box of the size $5 d_{s}$ concentric with the particle of concern is used as the local control volume. The equations for the Sherwood number and the Nusselt number calculation are the same as the Equation (36) and (37) respectively, with the driving force redefined locally using the value obtained from the control volume instead of the value at the inlet:

$$
\phi_{f, \text { local }}=\frac{\iiint_{V_{f}} g(r) \phi_{f, p} d V}{\iiint_{V_{f}} g(r) d V}
$$

In this equation, $g(r)=\exp \left[-r / R_{s}\right]$ is a weighing function of the distance between any fluid point inside the local control volume and the center of the reference particle. In Fig. 13 the distributions of the particle based Sherwood and Nusselt numbers are shown. A very significant range of scatter is depicted in both plots. The reason 

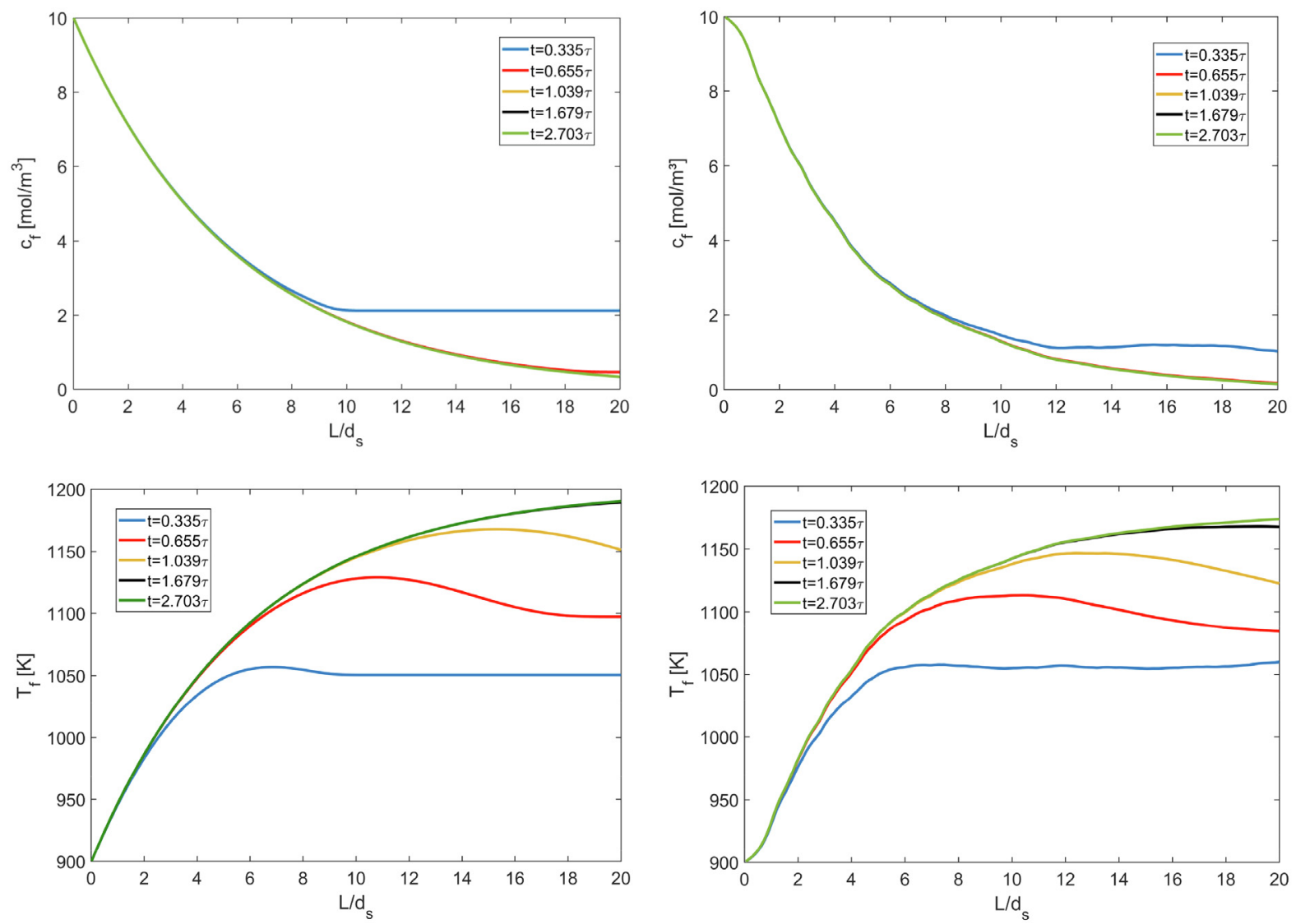

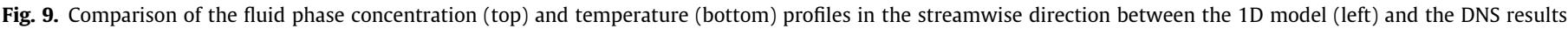
(right) at five intermediate time moments at the particle Reynolds number of 160 .

for this variation is the same as the earlier reported varying solid temperatures, namely the existence of highly irregular local flow fields. We also tried to correlate the mass and heat transfer rates of individual particles with the local fluid velocity, namely the Reynolds number for a certain control volume surrounding the reference particle. Volume size, relative position between particle and control volume, weighing method as well as the location of effective velocities were examined to select a proper local Reynolds number. However, for all cases the correlation coefficient was found to be very small, indicating no correspondence between these two quantities. An example is given in Fig. 14 that the Nusselt number of individual particles is marked in the flow field $\left(R e_{s}=160\right)$ in both streamwise and lateral directions. A cubic box of the lateral domain size is clipped in the middle part of the particle array, and two central planes of this box are sliced. In both plots, high values of the Nusselt number do not predominantly occur in regions with high local fluid velocity, meanwhile some particles there possess comparatively low Nusselt numbers. The same transversal crosssection is plotted at different Reynolds numbers in Fig. 15. Although the Nusselt number of most particles increases at higher Reynolds numbers, the increased amount varies significantly. It is more interesting to notice that, for the particles experiencing the highest local fluid velocity (upper-right corner), both increased and decreased Nusselt numbers are obtained at higher Reynolds numbers. All these factors reveal that the local interfacial transfer behavior depends on more parameters than simply the local fluid velocity. Looking at the entire distribution, both Sherwood and Nusselt numbers shift to the right with higher Reynolds numbers, with the probability of large values (right side) increased to some extent. As expected from the Colburn analogy, the distributions of the Sherwood number and the Nusselt number are very similar. The left side of the Nusselt number distribution is slightly higher, and this results in a lower mean value. As listed in Table 4, the mean values agree well with the empirical values predicted by the Gunn correlation. It should be noted that the overall mass and heat transfer behavior correlates well with the overall fluid Reynolds number, although such correlation is not observed in any local cases. The standard deviation $\sigma$, skewness $\mathcal{S}$ and kurtosis $\mathcal{K}$ are computed by the following three equations and listed in Table 4 as well.

$\sigma=\sqrt{\frac{\sum_{i=1}^{N_{s}}\left(X_{s, i}-\bar{X}_{s}\right)}{N_{s}-1}}$

$\mathcal{S}=\frac{\sum_{i=1}^{N_{s}}\left(X_{s, i}-\bar{X}_{s}\right)^{3}}{N_{s} \sigma^{3}}$

$\mathrm{K}=\frac{\sum_{i=1}^{N_{s}}\left(X_{s, i}-\bar{X}_{s}\right)^{4}}{N_{s} \sigma^{4}}$

In above equations, $X_{s, i}$ is the Sherwood or Nusselt number of individual particles, $\bar{X}_{s}$ is the corresponding mean value and $N_{s}$ is the total number of particles. The ratio of the standard deviation to the mean value decreases for both Sherwood and Nusselt numbers with higher Reynolds numbers, indicating a more uniform interfacial transfer performance. The skewness and kurtosis are computed here to indicate the deviation between the current Sherwood or Nusselt number distributions and a normal distribution. For a normal distribution, the values of the skewness and kurtosis are 0 and 3 , respectively. In the scatters of the Sherwood or Nusselt number 

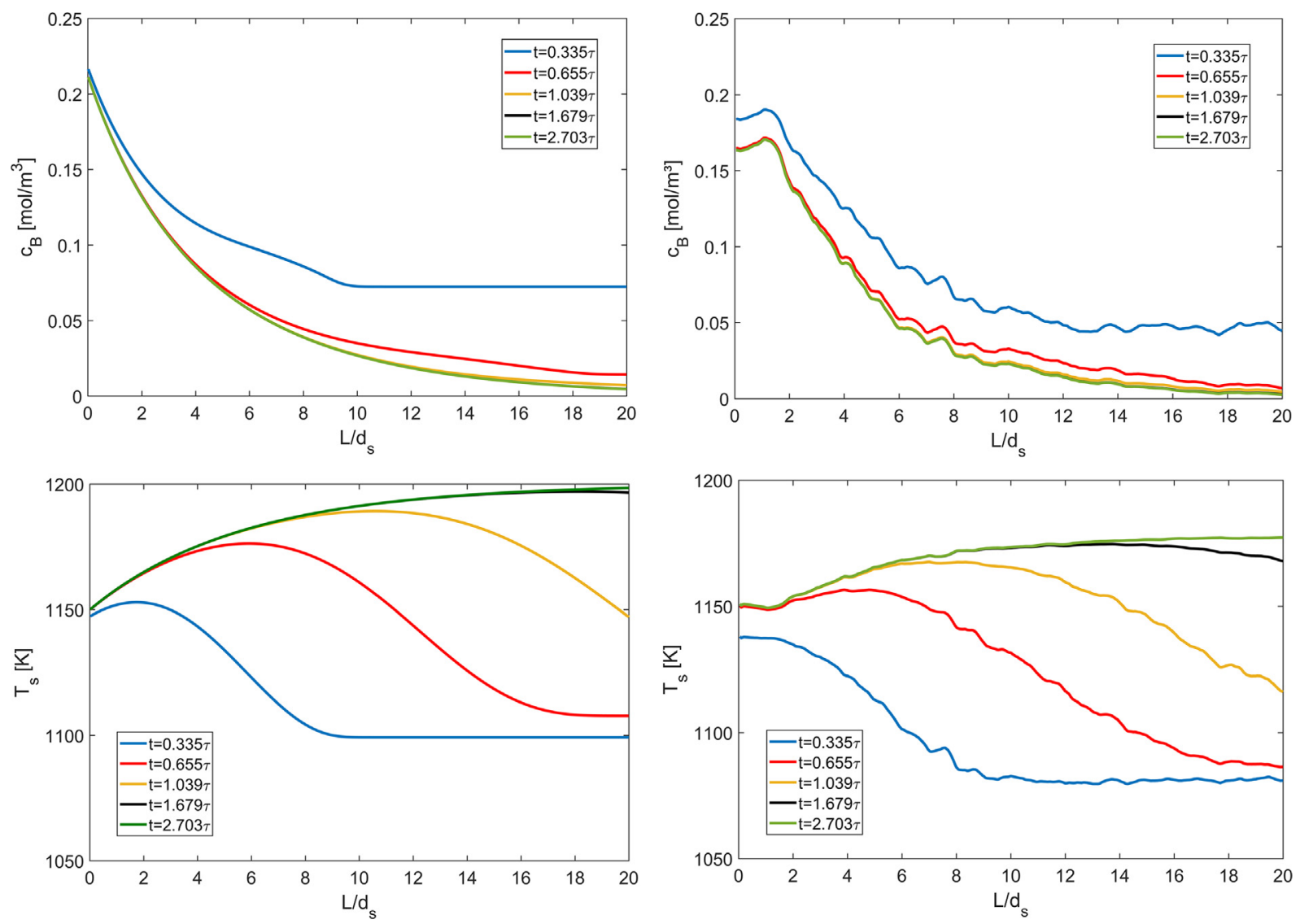

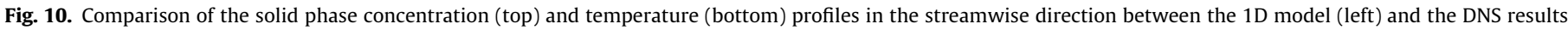
(right) at five intermediate time moments at the particle Reynolds number of 160 .
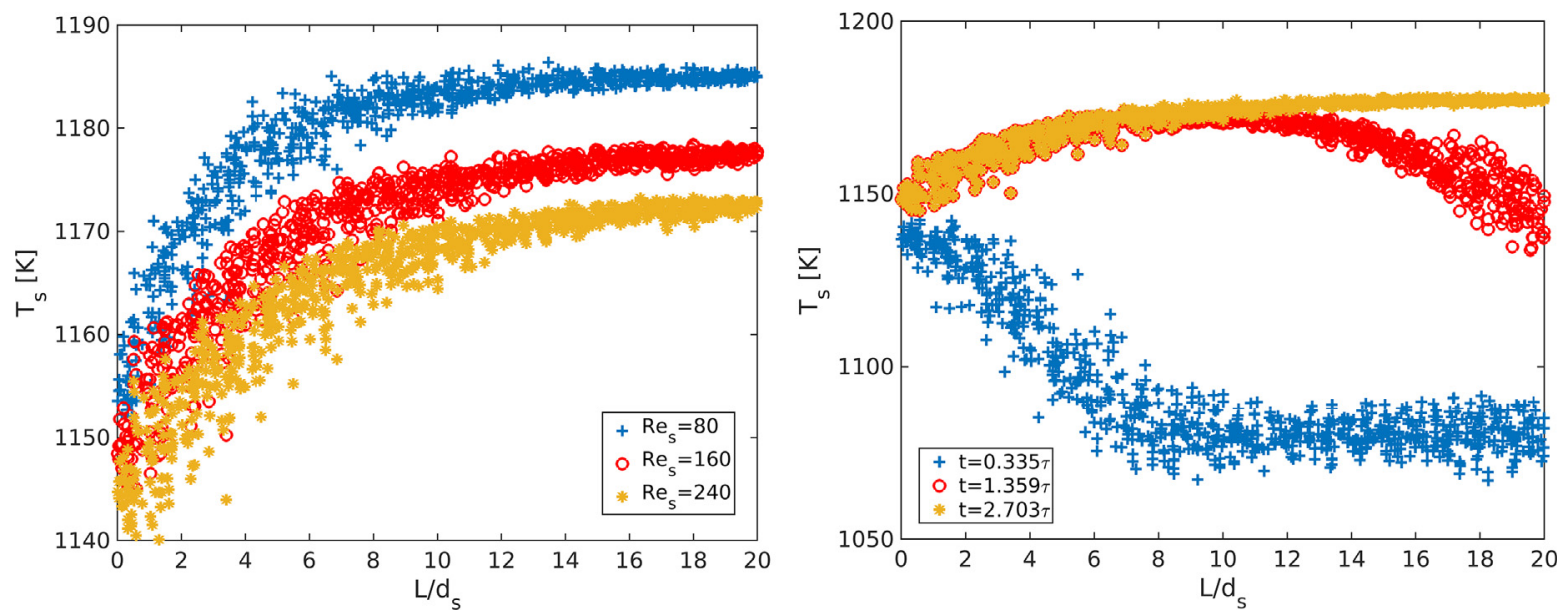

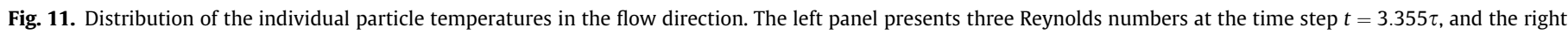
panel presents three intermediate time steps at the particle Reynolds number of 160 .

these two values, being around 1 and 5 respectively, suggest that the distributions are right-skewed with heavier tails compared with a normal distribution. With higher Reynolds numbers, both of them show closer distributions towards the mean value for both the Sherwood and Nusselt numbers. The difference of these two parameters between Sherwood and Nusselt numbers are thought due to the different boundary conditions, especially the influence of varying reaction rate of individual particles.

\section{Conclusions and outlook}

In this paper, we applied a DNS-IBM model to study integrated reactive fluid-particle systems. The IBM is based on a ghost-cell approach. A quadratic interpolation scheme is constructed to obtain the required ghost values and subsequently enters the discretized fluid phase governing equations. For the mass transfer process a first order exothermic reaction is assumed to proceed 

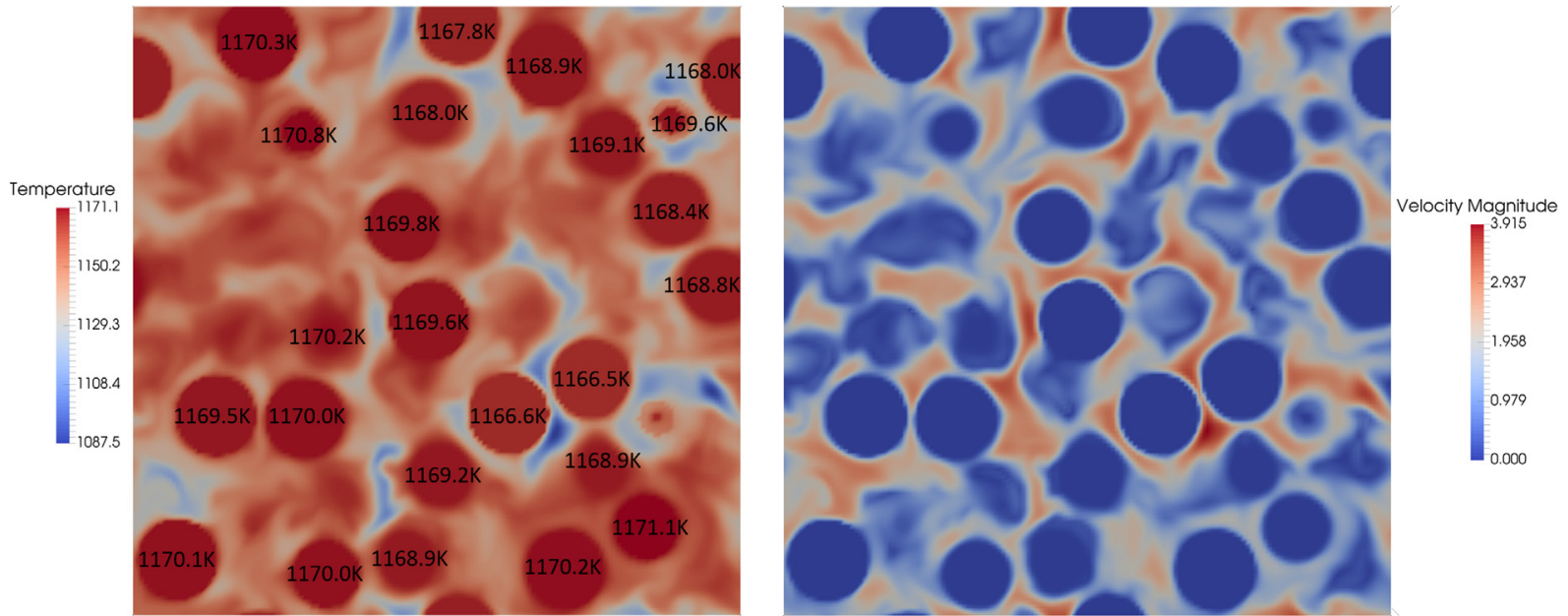

Fig. 12. Visualization of the temperature (left) and velocity (right) fields. Snapshots are shown for the cross-section in the radial direction at the packing height of $0.055 \mathrm{~m}$ at $t=3.292 \tau$ at the particle Reynolds number of 240 .
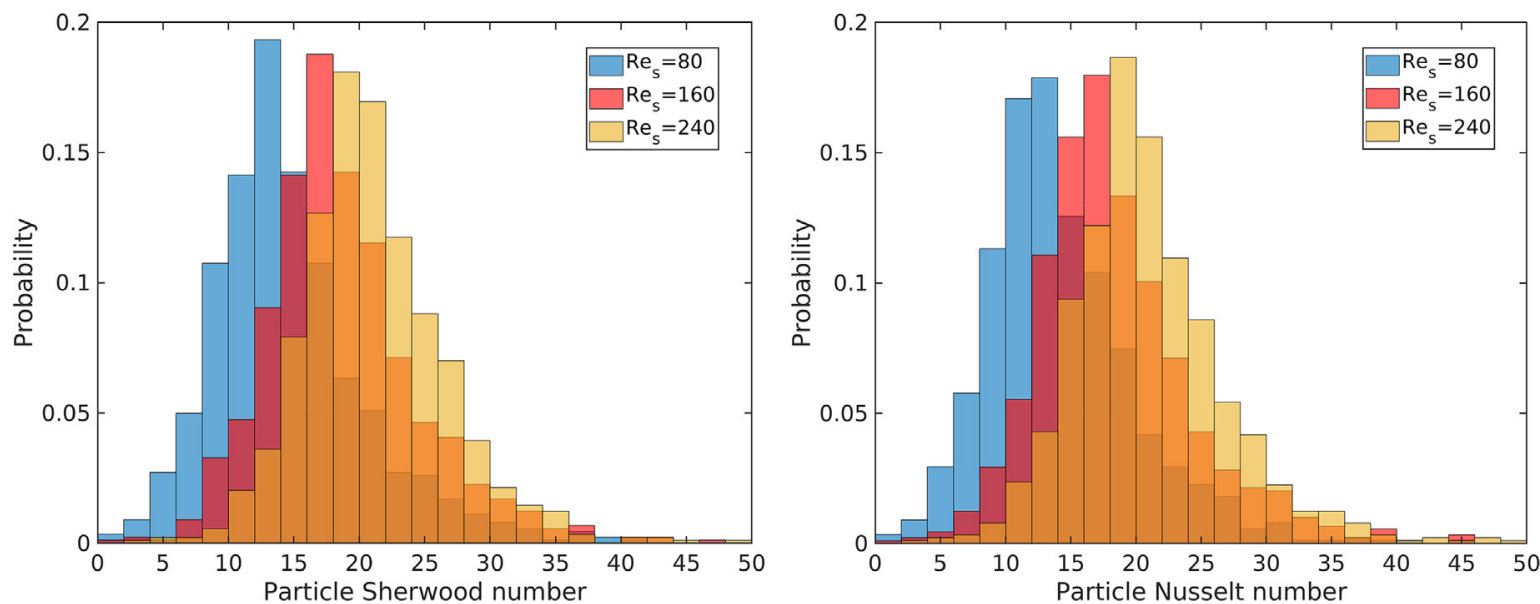

Fig. 13. Distribution of the particle Sherwood number (left) and the Nusselt number (right) computed using the local control volume.
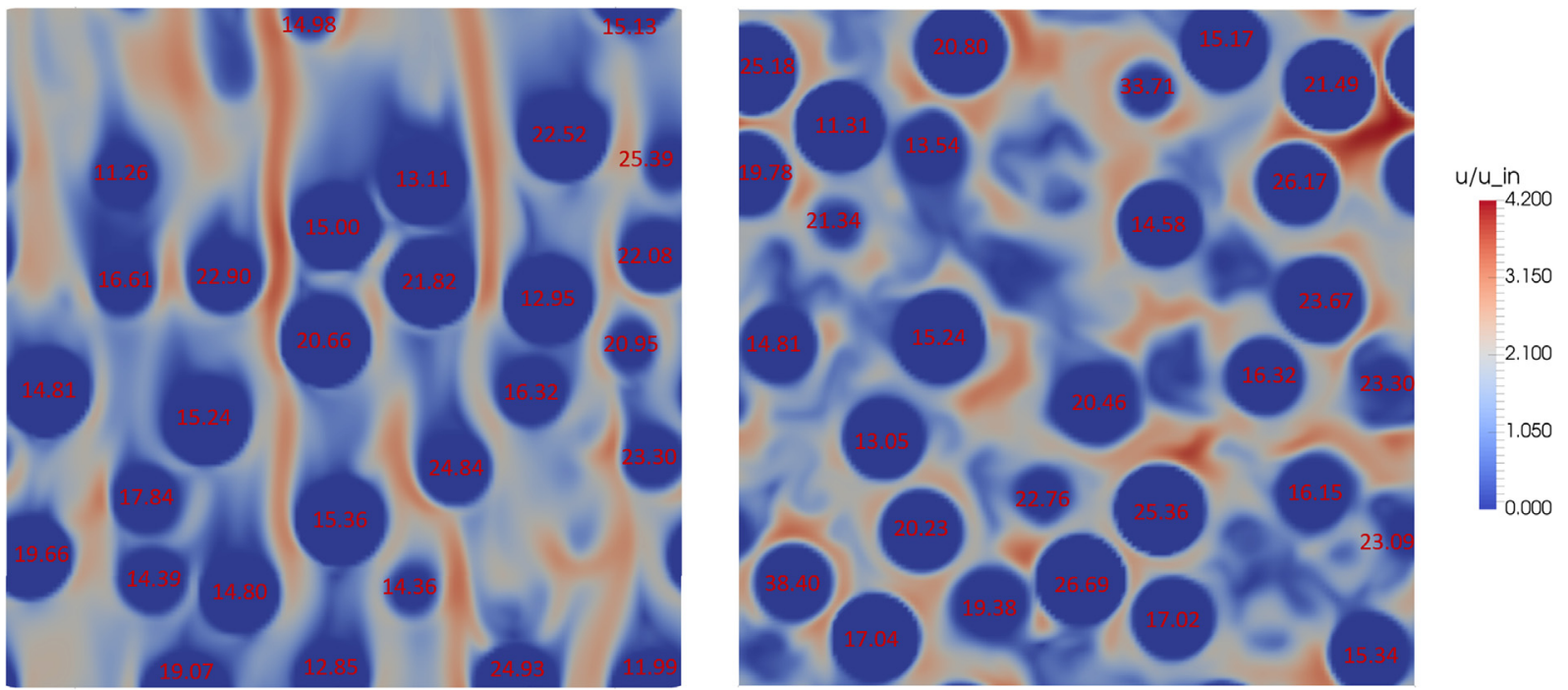

Fig. 14. Distribution of the Nusselt number of individual particles in the longitudinal $(0.045<x<0.0825)$ and transversal $(x=0.06)$ cross-sections at the particle Reynolds number of 160 (left panel with the flow direction from bottom to top and right panel with the flow direction into the paper, respectively). 

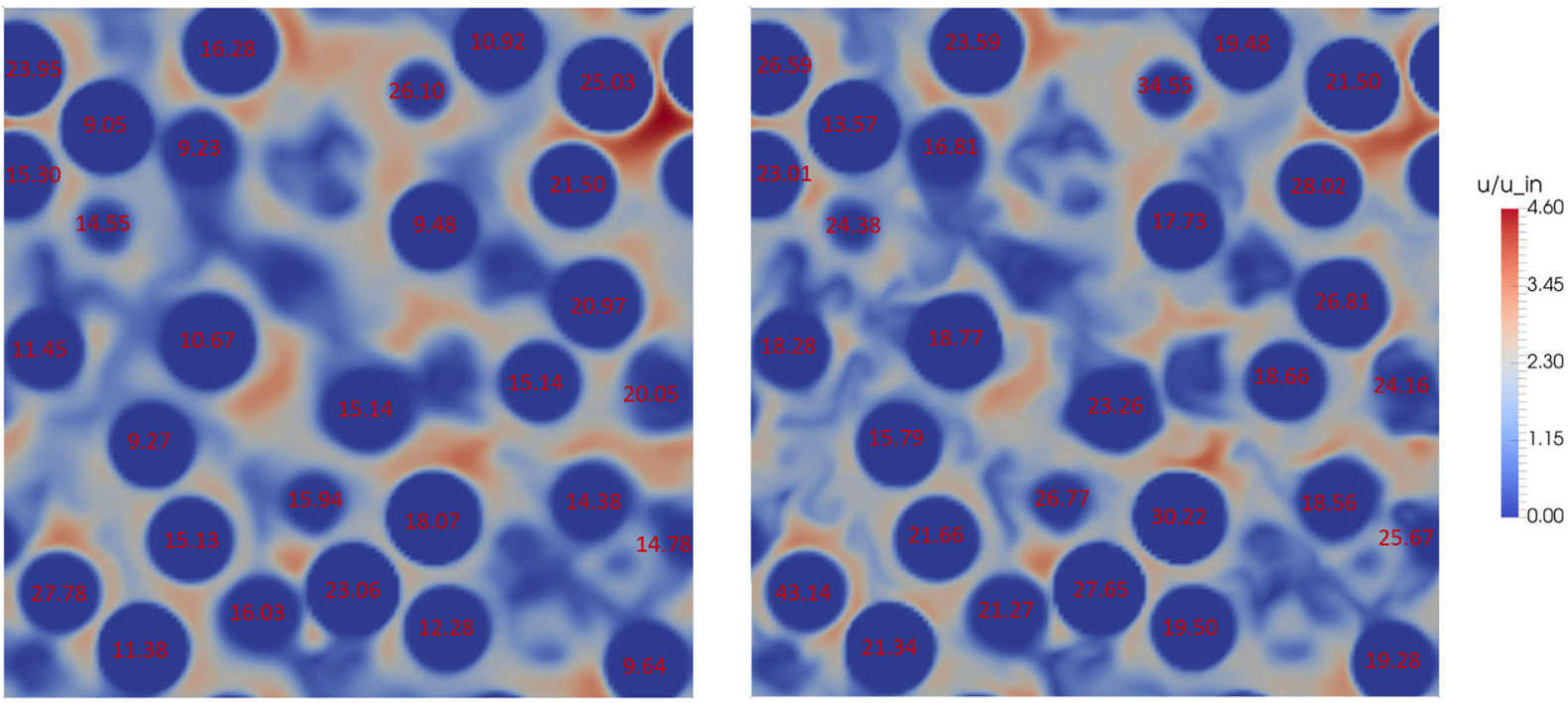

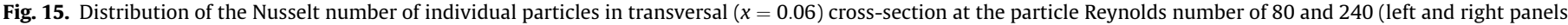
respectively, with the flow direction into the paper).

Table 4

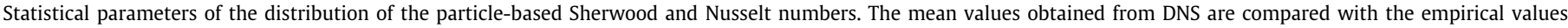
computed using Gunn correlation.

\begin{tabular}{|c|c|c|c|c|c|c|}
\hline$R e_{s}$ & $\mathrm{Sh} / \mathrm{Nu}$ & Gunn & DNS & $\sigma / \bar{X}$ & $\mathcal{S}$ & $\mathcal{K}$ \\
\hline \multirow[t]{2}{*}{80} & $S h$ & 14.43 & 14.63 & 0.40 & 1.04 & 4.89 \\
\hline & $\mathrm{Nu}$ & 13.59 & 14.18 & 0.40 & 1.08 & 5.46 \\
\hline \multirow[t]{2}{*}{160} & $S h$ & 19.45 & 18.70 & 0.31 & 0.86 & 4.79 \\
\hline & $N u$ & 18.25 & 18.34 & 0.33 & 1.07 & 5.65 \\
\hline \multirow[t]{2}{*}{240} & $S h$ & 23.59 & 21.13 & 0.27 & 0.83 & 5.65 \\
\hline & $\mathrm{Nu}$ & 22.10 & 20.99 & 0.29 & 1.09 & 6.57 \\
\hline
\end{tabular}

on the catalyst surface with a temperature-dependent reaction rate, while for the heat transfer process catalysts are heated up by the liberated reaction heat and consequently transfer the thermal energy to the fluid phase. In other words, the heat and mass transfer are strongly coupled by the highly non-linear Arrhenius equation.

We report three fluid-particle systems with increasing physical complexity, and to make our simulations closer to industrial applications, realistic parameters from the process of partial oxidation of methane are used. For the single particle system, the solid temperature shows some discrepancies between the DNS results and the values predicted by using the empirical Sherwood and Nusselt numbers, although for the Sherwood and Nusselt numbers themselves DNS results and empirical values are in a good agreement. This high sensitivity results from the strongly coupled heat and mass transfer processes. For the three particles system, the solid temperature is determined by the interplay between liberated reaction heat and convective heat transfer, and the contribution of individual particle to reactant conversion as well as temperature rise decreases from 1 st to 3 rd particle. For the dense array system, the time step is critical for the simulations due to the stiff heat and mass coupling in such a dense particle array. A higher Reynolds number results in slower reactant conversion and less temperature rise. The transient behavior of mass and heat transfer processes are assessed, and the profiles of the concentration and temperature in both fluid and solid phases are compared with a one-dimensional heterogeneous model. Qualitatively good agreement is reached, with the main difference that lower temperatures are obtained from DNS. The local heterogeneity is further studied by the solid temperature as well as the Sherwood and Nusselt numbers of indi- vidual particles. Significant variations are observed inside the array, however no correlation with local isotropic quantities could be obtained. Nevertheless, the average Sherwood and Nusselt numbers agree well with the values obtained from the empirical equations, i.e., a good correlation for the overall behavior is obtained.

Further work will certainly focus on the application of the current DNS model in industrial processes. For this purpose, the external transport of multicomponent reactant and product mixtures, polydispersed particle sizes, realistic reaction kinetics as well as the transport inside porous catalysts need to be taken into account. Besides that, good numerical stability will be beneficial in solving strongly non-linear heat and mass coupling. For that, internal iterations with a time-step adaption might be required. Using massive simulations, the performance of a real reaction process can be predicted. This will provide detailed information for its further optimization.

\section{Conflict of interest}

The authors declare that they have no known competing financial interests or personal relationships that could have appeared to influence the work reported in this paper.

\section{Acknowledgements}

This work was supported by the Netherlands Center for Multiscale Catalytic Energy Conversion (MCEC), an NWO Gravitation programme funded by the Ministry of Education, Culture and Science of the government of the Netherlands. 


\section{This work was carried out on the Dutch national e- infrastructure with the support of SURF Cooperative.}

\section{References}

Abdelsamie, A., Fru, G., Oster, T., Dietzsch, F., Janiga, G., Thévenin, D., 2016. Towards direct numerical simulations of low-Mach number turbulent reacting and twophase flows using immersed boundaries. Comput. Fluids 131, 123-141.

Akiki, G., Jackson, T.L., Balachandar, S., 2016. Force variation within arrays of monodisperse spherical particles. Phys. Rev. Fluids 1.

Boukharfane, R., Eugênio Ribeiro, F.H., Bouali, Z., Mura, A., 2018. A combined ghostpoint-forcing / direct-forcing immersed boundary method (IBM) for compressible flow simulations. Comput. Fluids 162, 91-112.

Chesshire, G., Henshaw, W.D., 1990. Composite overlapping meshes for the solution of partial differential equations. J. Comput. Phys. 90, 1-64.

Das, S., Deen, N.G., Kuipers, J.A.M., 2017. Immersed boundary method (IBM) based direct numerical simulation of open-cell solid foams: Hydrodynamics. AIChE J. $63,1152-1173$

Deen, N.G., Kriebitzsch, S.H.L., van der Hoef, M.A., Kuipers, J.A.M., 2012. Direct numerical simulation of flow and heat transfer in dense fluid-particle systems. Chem. Eng. Sci. 81, 329-344.

Deen, N.G., Kuipers, J.A.M., 2013. Direct numerical simulation of fluid flow and mass transfer in dense fluid-particle systems. Ind. Eng. Chem. Res. 52, 11266-11274.

Deen, N.G., Kuipers, J.A.M., 2014. Direct numerical simulation of fluid flow accompanied by coupled mass and heat transfer in dense fluid-particle systems. Chem. Eng. Sci. 116, 645-656.

Di, S., Ge, W., 2015. Simulation of dynamic fluid-solid interactions with an improved direct-forcing immersed boundary method. Particuology 18, 22-34.

Dixon, A.G., 2017. Local transport and reaction rates in a fixed bed reactor tube: Endothermic steam methane reforming. Chem. Eng. Sci. 168, 156-177.

Fadlun, E.A., Verzicco, R., Orlandi, P., Mohd-Yusof, J., 2000. Combined immersedboundary finite-difference methods for three-dimensional complex flow simulations. J. Comput. Phys. 161, 35-60.

Feng, Z.-G., Michaelides, E.E., 2000. A numerical study on the transient heat transfer from a sphere at high Reynolds and Peclet numbers. Int. J. Heat Mass Transf. 43, 219-229.

Feng, Z.-G., Michaelides, E.E., 2009. Heat transfer in particulate flows with Direct Numerical Simulation (DNS). Int. J. Heat Mass Transf. 52, 777-786.

Ghias, R., Mittal, R., Dong, H., 2007. A sharp interface immersed boundary method for compressible viscous flows. J. Comput. Phys. 225, 528-553.

Goldstein, D., Handler, R., Sirovich, L., 1993. Modeling a no-slip flow boundary with an external force field. J. Comput. Phys. 105, 354-366.

Gong, X., Gong, Z., Huang, H., 2014. An immersed boundary method for mass transfer across permeable moving interfaces. J. Comput. Phys. 278, 148-168.

Groote, A.M.D., Froment, G.F., 1996. Simulation of the catalytic partial oxidation of methane to synthesis gas. Appl. Catal. A 138, 245-264.

Gunn, D.J., 1978. Transfer of heat or mass to particles in fixed and fluidised beds. Int J. Heat Mass Transf. 21, 467-476.

Holloway, W., Sundaresan, S., 2012. Filtered models for reacting gas-particle flows. Chem. Eng. Sci. 82, 132-143.

Hu, H.H., Patankar, N.A., Zhu, M.Y., 2001. Direct numerical simulations of fluid-solid systems using the arbitrary Lagrangian-Eulerian technique. J. Comput. Phys. 169, 427-462.

Hughmark, G.A., 1980. Heat and mass transfer for spherical particles in a fluid field Ind. Eng. Chem. Fundam. 19, 198-201.

Jackson, R., 1997. Locally averaged equations of motion for a mixture of identical spherical particles and a Newtonian fluid. Chem. Eng. Sci. 52, 2457-2469.

Kim, J., Kim, D., Choi, H., 2001. An immersed-boundary finite-volume method for simulations of flow in complex geometries. J. Comput. Phys. 171, 132-150.

Kim, S.D., Kang, Y., 1997. Heat and mass transfer in three-phase fluidized-bed reactors-an overview. Chem. Eng. Sci. 52, 3639-3660.

Kravets, B., Kruggel-Emden, H., 2017. Investigation of local heat transfer in random particle packings by a fully resolved LBM-approach. Powder Technol. 318, 293 305.

Ku, X., Li, T., Løvås, T., 2015. CFD-DEM simulation of biomass gasification with steam in a fluidized bed reactor. Chem. Eng. Sci. 122, 270-283.

Kumar, S., Fan, L.S., 1994. Heat-transfer characteristics in viscous gas-liquid and gas-liquid-solid systems. AIChE Journal 40, 745-755.

Ladd, A.J.C., 1994a. Numerical simulations of particulate suspensions via discretized Boltzmann equation. Part 1. theoretical foundation. J. Fluid Mech. 271, 285-309.

Ladd, A.J.C., 1994b. Numerical simulations of particulate suspensions via a discretized Boltzmann equation. Part 2. numerical results. J. Fluid Mech. 271 311-339.
Link, J.M., Cuypers, L.A., Deen, N.G., Kuipers, J.A.M., 2005. Flow regimes in a spoutfluid bed: a combined experimental and simulation study. Chem. Eng. Sci. 60, 3425-3442.

Liu, C., Hu, C., 2014. An efficient immersed boundary treatment for complex moving object. J. Comput. Phys. 274, 654-680.

Lu, J., Das, S., Peters, E.A.J.F., Kuipers, J.A.M., 2018a. Direct numerical simulation of fluid flow and mass transfer in dense fluid-particle systems with surface reactions. Chem. Eng. Sci. 176, 1-18.

Lu, J., Peters, E.A.J.F., Kuipers, J.A.M., 2018b. Direct numerical simulation of fluid flow and mass transfer in particle clusters. Ind. Eng. Chem. Res. 57, 4664-4679.

Lu, J., Tan, M.D., Peters, E.A.J.F., Kuipers, J.A.M., 2018c. Direct Numerical Simulation of Reactive Fluid-Particle Systems Using an Immersed Boundary Method. Ind. Eng. Chem. Res. 57, 15565-15578.

Lu, J., Zhu, X., Peters, E.A.J.F., Verzicco, R., Lohse, D., Kuipers, J.A.M., 2018d. Moving from momentum transfer to heat transfer - a comparative study of an advanced Graetz-Nusselt problem using immersed boundary methods. Chem. Eng. Sci.

Marella, S., Krishnan, S., Liu, H., Udaykumar, H.S., 2005. Sharp interface Cartesian grid method I: an easily implemented technique for 3D moving boundary computations. J. Comput. Phys. 210, 1-31.

Melchiori, T., Di Felice, L., Mota, N., Navarro, R.M., Fierro, J.L.G., Annaland, M.v.S., Gallucci, F., 2014. Methane partial oxidation over a LaCr0.85Ru0.1503 catalyst: Characterization, activity tests and kinetic modeling. Appl. Catal. A 486, 239249.

Mittal, R., Dong, H., Bozkurttas, M., Najjar, F.M., Vargas, A., von Loebbecke, A., 2008. A versatile sharp interface immersed boundary method for incompressible flows with complex boundaries. J. Comput. Phys. 227, 4825-4852.

Mohd-Yusof, J., 1997. Combined Immersed-Boundary and B-spline methods for simulations of flow in complex geometries. Center Turbul. Res. Ann. Res. Brief, 317-327.

Peskin, C.S., 1977. Numerical analysis of blood flow in the heart. J. Comput. Phys. 25, $220-252$.

Richter, A., Nikrityuk, P.A., 2012. Drag forces and heat transfer coefficients for spherical, cuboidal and ellipsoidal particles in cross flow at sub-critical Reynolds numbers. Int. J. Heat Mass Transf. 55, 1343-1354.

Saiki, E.M., Biringen, S., 1996. Numerical simulation of a cylinder in uniform flow: application of a virtual boundary method. J. Comput. Phys. 123, 450-465.

Schulze, S., Nikrityuk, P., Compart, F., Richter, A., Meyer, B., 2017. Particle-resolved numerical study of char conversion processes in packed beds. Fuel 207, 655662 .

Takeuchi, S., Yuki, Y., Ueyama, A., Kajishima, T., 2010. A conservative momentumexchange algorithm for interaction problem between fluid and deformable particles. Int. J. Numer. Meth. Fluids 64, 1084-1101.

Tenneti, S., Sun, B., Garg, R., Subramaniam, S., 2013. Role of fluid heating in dense gas-solid flow as revealed by particle-resolved direct numerical simulation. Int. J. Heat Mass Transf. 58, 471-479.

Tseng, Y.-H., Ferziger, J.H., 2003. A ghost-cell immersed boundary method for flow in complex geometry. J. Comput. Phys. 192, 593-623.

Udaykumar, H.S., Mittal, R., Rampunggoon, P., Khanna, A., 2001. A sharp interface cartesian grid method for simulating flows with complex moving boundaries. J. Comput. Phys. 174, 345-380.

Uhlmann, M., 2005. An immersed boundary method with direct forcing for the simulation of particulate flows. J. Comput. Phys. 209, 448-476.

Validi, A., Schock, H., Jaberi, F., 2017. Turbulent jet ignition assisted combustion in a rapid compression machine. Combust. Flame 186, 65-82.

Vanella, M., Balaras, E., 2009. A moving-least-squares reconstruction for embeddedboundary formulations. J. Comput. Phys. 228, 6617-6628.

Wagner, I., Stichlmair, J., Fair, J.R., 1997. Mass transfer in beds of modern, highefficiency random packings. Ind. Eng. Chem. Res. 36, 227-237.

Wakao, N., Funazkri, T., 1978. Effect of fluid dispersion coefficients on particle-tofluid mass transfer coefficients in packed beds. Chem. Eng. Sci. 33, 1375-1384.

Wehinger, G.D., Eppinger, T., Kraume, M., 2015. Detailed numerical simulations of catalytic fixed-bed reactors: Heterogeneous dry reforming of methane. Chem. Eng. Sci. 122, 197-209.

Xia, J., Luo, K., Fan, J., 2015. Simulating heat transfer from moving rigid bodies using high-order ghost-cell based immersed-boundary method. Int. J. Heat Mass Transf. 89, 856-865.

Xu, Y., Padding, J.T., van der Hoef, M.A., Kuipers, J.A.M., 2013. Detailed numerical simulation of an intruder impacting on a granular bed using a hybrid discrete particle and immersed boundary (DP-IB) method. Chem. Eng. Sci. 104, 201-207.

Xue, Q., Fox, R.O., 2014. Multi-fluid CFD modeling of biomass gasification in polydisperse fluidized-bed gasifiers. Powder Technol. 254, 187-198.

Zhuang, Y.-Q., Chen, X.-M., Luo, Z.-H., Xiao, J., 2014. CFD-DEM modeling of gas-solid flow and catalytic MTO reaction in a fluidized bed reactor. Comput. Chem. Eng. $60,1-16$. 